\title{
Multiphysics Lattice Discrete Particle Model for the Simulation of Concrete Thermal Spalling
}

\author{
Lei Shen ${ }^{\mathrm{a}, \mathrm{b}}$, Weixin Li ${ }^{\mathrm{b}}$, Xinwei Zhou ${ }^{\mathrm{c}}$, Jun Feng ${ }^{\mathrm{b}, \mathrm{d}}$, Giovanni Di Luzio ${ }^{\mathrm{e}}$, \\ Qingwen Ren ${ }^{\mathrm{a}}$, Gianluca Cusatis ${ }^{\mathrm{b}}$ \\ ${ }^{a}$ Hohai University, Department of Engineering Mechanics, Nanjing, 210098, China; \\ ${ }^{b}$ Northwestern University, Department of Civil and Environmental Engineering, \\ Evanston, IL, 60208, USA; \\ ${ }^{c}$ ES3, San Diago, CA, 92101, USA; \\ ${ }^{d}$ Nanjing University of Science and Technology, National Key Laboratory of Transient \\ Physics, Nanjing, 210094, China; \\ ${ }^{e}$ Politecnico di Milano, Department of Civil and Environmental Engineering, Milan, \\ 20121, Italy.
}

\begin{abstract}
Explosive thermal spalling behavior during fire exposure is one of the major issues in the design of modern reinforced concrete structures. Previous experience on fire disasters indicates that spalling of concrete can have serious structural and economic consequences and must be taken into account in the design for fire. However, spalling mechanisms and their interaction still remain in dispute in the scientific community. In order to shed some light on this phenomenon, a discrete hygro-thermal model of concrete at high temperature called DTemPor3 is proposed and a full coupling scheme between DTemPor3 and the Lattice Discrete Particle Model (LDPM) is performed. The proposed multi-physical coupled model features the effect of pore pressure and temperature on the mechanical response as well as the impact of
\end{abstract}

\footnotetext{
*Corresponding author

Email address: g-cusatis@northwestern.edu (Gianluca Cusatis)
} 
cracking on moisture mass transport and heat transfer. Simulations of typical spalling experiments show good agreements with data gathered from the literature for both high-performance concrete and ordinary concrete, demonstrating the accuracy of the proposed approach. Cracking localization is found to significantly impede the local pore pressure build-up due to the increase of pore or crack volume. The numerical simulations demonstrate that the spalling phenomenon can be successfully reproduced, only when the effect of thermal stresses is taken in account along with the effect of pore pressure on crack initiation.

Keywords: concrete thermal spalling, high temperature, lattice discrete particle model, hygro-thermal coupling

1. Introduction

2 Concrete tends to be very sensitive to explosive spallling when it is ex3 posed to fire and its sensitivity to this phenomenon must be considered and 4 controlled in the design and construction of reinforced concrete structures, 5 e.g. for the design of tunnels. Explosive spalling can lead to a significant 6 reduction of the cross sectional area and to the possible direct exposure of 7 the reinforcing bars to the flames with a dramatical decay of the mechanical properties of steel. In addition, new types of concretes, e.g. high and ultrahigh strength, high-performance and self-compacting concretes, have shown an increased sensitivity to spalling due to fire exposure. For example, up to $75 \%$ and $100 \%$ of the concrete thickness was lost due to explosive spalling in the 1994 Great Belt tunnel fire and in the 1996 Channel tunnel fire, respectively. This was due to the fact that the high performance concretes 
used in these tunnels had a higher tendency to experience explosive spalling than normal strength concrete due to their denser and less porous structure. Furthermore, in the Mont Blanc Tunnel fire in 1999, a 900-m-long portion of the tunnel roof lining completely collapsed and exposed the rock.

Although thermal spalling of concrete has been a hot topic for the scientific community over the last 30 years, engineers still lack a complete understanding of the phenomenon and do not have properer tools for designing against this kind of failure. In fact, the main driving mechanisms are not completely clear due to the complex interaction of heat transfer, moisture mass transport, as well as mechanical and chemical behavior at high temperature. Fire or other severe thermal environmental conditions can cause extreme high temperature gradients, large increase of permeability, cement dehydration, and water phase changes in concrete. Internal stresses build up as a result of the combined effect of pore pressure increase, thermal stresses derived from temperature gradients, mismatch between the deformation of different constituents, and the shrinkage associated with water release. When the internal stresses exceed the maximum tensile strength, cracks and/or spalling occur.

Many experimental and numerical investigations have been conducted in the last decades to gain an understanding of the driving mechanisms on concrete spalling behavior, and it is now commonly agreed that the two main actors are: (1) the pore (vapor) pressure mechanism [? ? ? ], and (2) the thermal stress mechanism [? $\quad$ ? $\quad$ ? $\quad$ ? $\quad$ ? $\quad$ ? $\quad$ ? $]$. On the one hand, the pressure builds up in the concrete pores as a consequence of the physically and chemically bound water in the cement vaporizing at high temperature, and it leads to tensile stresses in the heated concrete as illustrated in Fig. 1(a). 

thermal spalling by means of accurate numerical calculations.

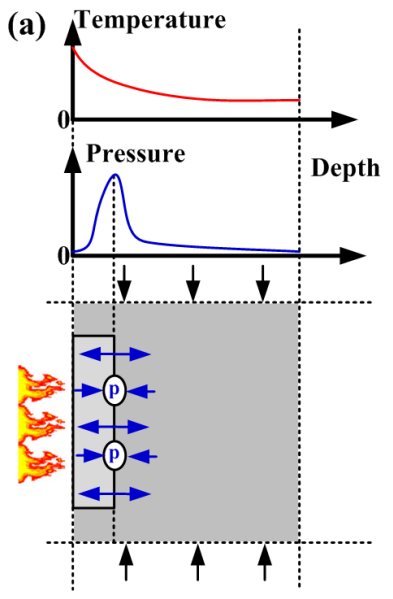

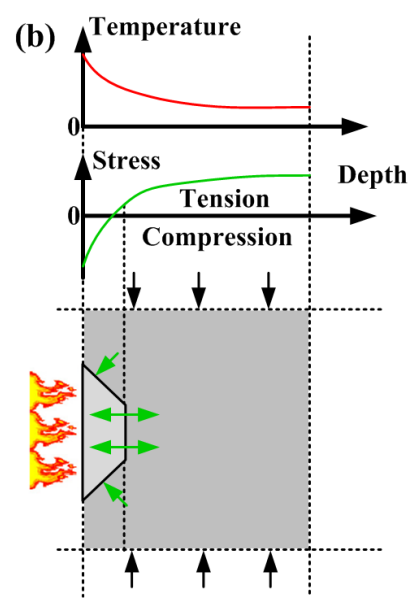
of stress with compressive stresses parallel to the heated surface and tensile stresses perpendicular to the heated surface as illustrated in Fig. 1(b). In the debate over spalling mechanisms, some researchers [? ? ? ] stand in a middle ground, and observe that spalling is a joint action of pore pressure build-up and elevated levels of elastic energy due to constrained thermal strains, rather than one of these factors acting alone. The objective of this study is to give a clear evidence for the main driving mechanisms of concrete

Figure 1: Spalling mechanisms: (a) pore (vapor) pressure; (b) thermal stress.

47

48

48

On the other hand, the restrained thermal dilation generate a biaxial state concrete exposed to high temperatures was inspired by the TemPor model [? ], in which mass balance of free water and conservation of energy were com1 bined to compute temperature and pore pressure distributions. The model 52 accounted for the release of chemically bound water due to dehydration and included desorption isotherms at elevated temperature developed by fitting 
experimental data. Later, based on the desorption isotherms of the TemPor model, Tenchev et al. [? ], Davie et al. [? ] and Dwaikat et al. [? ] developed their hygro-thermal models which took the gas phases (vapor and dry air) into consideration. Another remarkable model was presented by Gawin et al. [? ? ], in which they proposed a fully coupled hygro-thermal model using the Baroghel-Bouny et al. [? ]'s desorption isotherms and used capillary pressure as one of the primary variables instead of pore pressure.

Besides the development of hygro-thermal models, a coupled mechanical analysis is required to study spalling mechanisms. In most numerical studies [? ? ? ? ], continuous plastic and/or damage constitutive laws were adopted to model concrete cracking behavior. The main qualitative conclusion of those studies was that thermal stresses are the main energy source of thermal spalling while pore pressure is a triggering factor. Dwaikat et al. [? ] employed an engineering approach in which spalling was supposed to occur when the effective pore pressure exceeds the temperature dependent tensile strength of concrete. In this approach, the pore pressure was regarded as the driving factor for spalling.

Although the thermo-hygro-mechanical coupled behavior was accurately predicted by the traditional continuous finite element approaches and some reasonable discussions on the various mechanisms were given in previous studies, the dynamic explosive spalling phenomena was not reproduced vividly so far. Therefore, this study responds to an actual need for a reliable computational tool capable of simulating the dynamic explosive fragmentation during spalling.

In this numerical study, a discrete temperature and pore pressure model 
in three-dimension (called DTemPor3) is formulated and implemented within the framework of the Lattice Discrete Particle Model [? ? ]. Calibration and validation of the entire framework is conducted according to experiments available in the literature [? ]. The spalling phenomena observed in the experiments [? ] are reproduced and the main driving mechanisms are discussed.

\section{The Computational Framework}

\subsection{The Lattice Discrete Particle Model at high temperature}

The Lattice Discrete Particle Model (LDPM), originally formulated by Cusatis et al. [? ? ], is able to accurately capture the failure behavior of concrete at room temperature as demonstrated in many previous studies [? ? ? ? ? ? ? ? ? ? ? ]. This study proposes an improvement of LDPM in order to allow the simulation of concrete behavior at high temperature.

LDPM simulates the concrete mesostructure by taking into account the interaction of coarse aggregate pieces. The mesostructure is constructed through the following steps. (1) Coarse aggregate pieces approximated by spheres of different size are randomly placed inside the concrete volume. To mimic the real material mesostructure, the placement follows a particle size distribution curve consistent with a Fuller sieve curve. A typical aggregate distribution is shown in Fig. 2(a) in 2D and (d) in 3D. Over the external surfaces, zero-radius aggregate pieces (nodes) are randomly placed. (2) The Delaunay tetrahedralization is used to connect the centers (termed "nodes") of the spherical particles to produce tetrahedra. The edges of the tetrahedra form the lattice system that describes the interaction between adjacent 
(a)

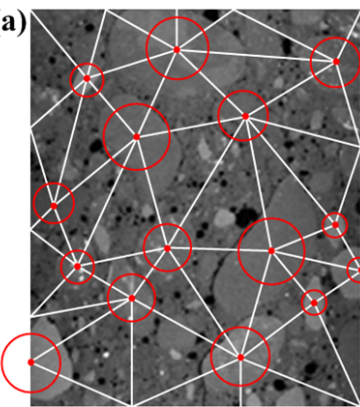

(d)

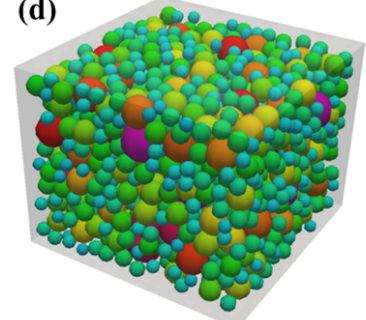

(b)

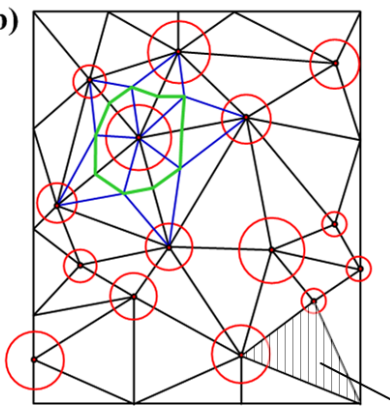

(e)

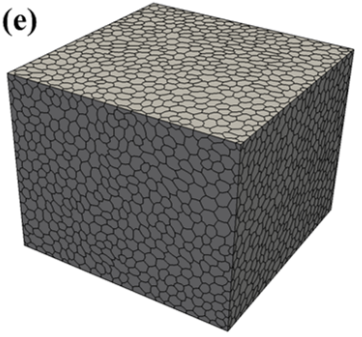

(c)

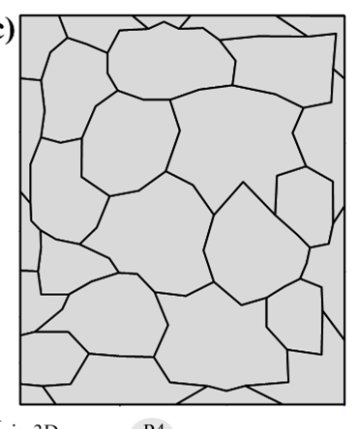

(f)

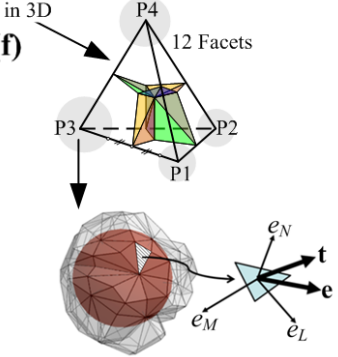

Figure 2: LDPM geometry. (a) Concrete mesostructure in 2D; (b) Delaunay triangulation in 2D; (c) LDPM cells in 2D; (d) LDPM particles in 3D; (e) LDPM cells in 3D; (f) LDPM facets.

If $\mathbf{x}_{i}$ and $\mathbf{x}_{j}$ are the positions of nodes $i$ and $j$ adjacent to a generic facet, 
the facet strains are defined as:

$$
\mathbf{e}=\left[\begin{array}{lll}
e_{N} & e_{M} & e_{L}
\end{array}\right]^{\mathrm{T}}=\left[\frac{\mathbf{n}^{\mathrm{T}} \llbracket \mathbf{u} \rrbracket}{l} \frac{\mathbf{m}^{\mathrm{T}} \llbracket \mathbf{u} \rrbracket}{l} \frac{\mathbf{l}^{\mathrm{T}} \llbracket \mathbf{u} \rrbracket}{l}\right]^{\mathrm{T}}
$$

where $e_{N}$ is the normal strain component, $e_{M}$ and $e_{L}$ are the tangential strain components, $\llbracket \mathbf{u} \rrbracket=\mathbf{u}_{j}-\mathbf{u}_{i}$ is the displacement jump at the centroid of the facet, $l=\left\|\mathbf{x}_{j}-\mathbf{x}_{i}\right\|_{2}$ is the distance between the two nodes, $\mathbf{n}=\left(\mathbf{x}_{j}-\mathbf{x}_{i}\right) / l$, and $\mathbf{m}, \mathbf{l}$ are two unit vectors mutually orthogonal and orthogonal to $\mathbf{n}$. The displacements $\mathbf{u}_{i}$ and $\mathbf{u}_{j}$ are calculated as a function of translational and rotational degrees of freedom of the particles through rigid body kinematics. These measures of strains are fully consistent with the classical definition of strains in continuum mechanics [? ? ? ? ? ? ? ]. By assuming additivity of strains, one can write:

$$
\mathbf{e}=\mathbf{e}^{s}+\mathbf{e}^{0}
$$

where $\mathbf{e}^{s}$ represents stress-related strains and $\mathbf{e}^{0}$ represents eigenstrains which can be caused by thermal expansion and shrinkage. The strain $\mathbf{e}^{s}$ depends on the tractions $\mathbf{t}^{s}$ acting on the solid skeleton through a vectorial constitute equation $\mathbf{t}^{s}=\mathbf{f}\left(\mathbf{e}^{s}\right)$. At high temperature, since the thermal strain is the most important eigenstrain component, one can write $\dot{\mathbf{e}}^{0}=\left[\begin{array}{lll}\alpha_{T} \dot{T} & 0 & 0\end{array}\right]^{\mathrm{T}}$, in which $\dot{T}$ is the temperature rate and $\alpha_{T}(T)$ is the thermal expansion coefficient, which is actually an overall result of the cement shrinkage and aggregate dilatation caused by the chemical and physical reactions at high temperature [? ]. $\alpha_{T}(T)$ is defined as a linear monotone increasing function with a minimum of $7 \times 10^{-6} 1 /{ }^{\circ} \mathrm{C}$ at room temperature and a maximum of $10 \times 10^{-6} 1 /{ }^{\circ} \mathrm{C}$ at $1000{ }^{\circ} \mathrm{C}$. Over $1000{ }^{\circ} \mathrm{C}$ concrete is considered to be melted and $\alpha_{T}=0$ [? ? ]. 
Load induced thermal strain (LITS) [? ] is the strain that develops when concrete is heated under load and includes basic creep, drying creep and transitional thermal creep as well as changes in the elastic parameters that occur during the heating process. The combination of transitional thermal creep and drying creep is often called transient creep and is by far the largest strain component of unsealed concrete heated to high temperatures. In this model LITS is modeled through the degradation of the LDPM elastic parameters as it will be discussed latter.

The LDPM elastic behavior is described by assuming that the normal and shear tractions acting on the solid skeleton are proportional to the corresponding strains:

$$
\mathbf{t}^{s}=\left[\begin{array}{lll}
t_{N}^{s} & t_{M}^{s} & t_{L}^{s}
\end{array}\right]^{\mathrm{T}}=\left[\begin{array}{lll}
E_{N} e_{N}^{s} & E_{T} e_{M}^{s} & E_{T} e_{L}^{s}
\end{array}\right]^{\mathrm{T}}
$$

where $t_{N}^{s}$ is the normal component, $t_{M}^{s}$ and $t_{L}^{s}$ are the shear components; $E_{N}=E_{0}, E_{T}=\alpha E_{0}, E_{0}$ is the effective normal elastic modulus which can be estimated as $E /(1-2 \nu)$ where $E$ is Young's modulus and $\nu$ is Poisson's ratio; $\alpha$ is the shear-normal coupling parameter and $\alpha=0.25$ for most concretes $[?]$.

In order to describe the inelastic behavior the constitutive relations on the facets are formulated as follows.

Fracturing and cohesive behavior under tension and tension/shear is simulated to occur for $e_{N}^{s}>0$. By defining the effective strain and the effective stress as $e^{s}=\left[e_{N}^{s 2}+\alpha\left(e_{M}^{s 2}+e_{L}^{s 2}\right)\right]^{1 / 2}$ and $t^{s}=\left[t_{N}^{s 2}+\left(t_{M}^{s 2}+t_{L}^{s 2}\right) / \alpha\right]^{1 / 2}$, one can write the relationship between tractions and stresses, and strains through damage-type constitutive equations as $t_{N}^{s}=t^{s} e_{N}^{s} / e^{s}, t_{M}^{s}=\alpha t^{s} e_{M}^{s} / e^{s}$ and $t_{L}^{s}=\alpha t^{s} e_{L}^{s} / e^{s}$ 
The effective stress $t^{s}$ is incrementally elastic $\dot{t}^{s}=E_{N} \dot{e}^{s}$ and limited by a strain-dependent boundary $0 \leqslant t^{s} \leqslant \sigma_{b t}\left(e^{s}, \omega\right)$ in which $\sigma_{b t}\left(e^{s}, \omega\right)=$ $\sigma_{0}(\omega) \exp \left[-H_{0}(\omega)\left\langle e_{\max }^{s}-e_{0}^{s}(\omega)\right\rangle / \sigma_{0}(\omega)\right],\langle x\rangle=\max (x, 0), \omega$ is the degree of interaction between shear and normal loading defined through $\tan (\omega)=$ $e_{N}^{s} /\left(\sqrt{\alpha} e_{T}^{s}\right)=t_{N}^{s} \sqrt{\alpha} / t_{T}^{s}$. $e_{T}^{s}$ is the total shear strain defined as $e_{T}^{s}=\left(e_{M}^{s 2}+\right.$ $\left.e_{L}^{s 2}\right)^{1 / 2}$ and $t_{T}^{s}$ is the total shear stress defined as $t_{T}^{s}=\left(t_{M}^{s 2}+t_{L}^{s 2}\right)^{1 / 2}$. The maximum effective strain is time dependent and is defined as $e_{\max }^{s}(\tau)=\left(e_{N, \max }^{s 2}+\right.$ $\left.\alpha e_{T, \text { max }}^{s 2}\right)^{1 / 2}$ where $e_{N, \max }^{s}(\tau)=\max _{\tau^{\prime}<\tau}\left[e_{N}^{s}\left(\tau^{\prime}\right)\right]$ and $e_{T, \max }^{s}(\tau)=\max _{\tau^{\prime}<\tau}\left[e_{T}^{s}\left(\tau^{\prime}\right)\right]$. The strength limit of the effective stress provides a smooth transition between pure tension $(\omega=\pi / 2)$ and pure shear $(\omega=0)$ and it is defined as $\sigma_{0}(\omega)=\sigma_{t}\left\{-\sin (\omega)+\left[\sin ^{2}(\omega)+4 \alpha \cos ^{2}(\omega) / r_{s t}^{2}\right]^{1 / 2}\right\} /\left[2 \alpha \cos ^{2}(\omega) r_{s t}^{2}\right]$ where $r_{s t}=\sigma_{s} / \sigma_{t}$ is the shear to tensile strength ratio. The post-peak softening modulus is formulated by a power function called the effective softening modulus $H_{0}(\omega)=H_{t}(2 \omega / \pi)^{n_{t}}$. Under pure tension, $H_{0}(\pi / 2)=H_{t}=$ $2 E_{0} /\left(l_{t} / l-1\right)$ where $H_{t}$ is the softening modulus, $n_{t}=0.2$ is the softening exponent, $l_{t}=2 E_{0} G_{t} / \sigma_{t}^{2}$ is the tensile characteristic length, $G_{t}$ is the mesoscale fracture energy, and $\sigma_{t}$ is the tensile strength.

The second set of equations describes pore collapse and material compaction in compression occurring for $e_{N}^{s}<0$. The strain hardening plastic behavior due to high compressive hydrostatic deformation is described through a strain-dependent boundary $\sigma_{b c}\left(e_{D}^{s}, e_{V}^{s}\right)$ at each facet which limits the normal compressive stress component through the inequality $-\sigma_{b c}\left(e_{D}^{s}, e_{V}^{s}\right) \leqslant$ $t_{N}^{s} \leqslant 0$, where $e_{V}^{s}=\Delta V_{s} / 3 V_{s 0}$ is the volumetric strain, computed as the change between the current and the initial volume of each LDPM tetrahedron. The volumetic strain $e_{V}^{s}$ is the same for all the facets of a given 
tetrahedron but the deviatoric strain $e_{D}^{s}=e_{N}^{s}-e_{V}^{s}$ varies from facet to facet. The strain-dependent boundary is defined for three different strain ranges: $\sigma_{b c}\left(e_{D}^{s}, e_{V}^{s}\right)=\sigma_{c 0}$ for $-e_{V}^{s} \leqslant 0 ; \sigma_{b c}\left(e_{D}^{s}, e_{V}^{s}\right)=\sigma_{c 0}+\left\langle-e_{V}^{s}-e_{c 0}\right\rangle H_{c}\left(r_{D V}\right)$ for $0 \leqslant-e_{V}^{s} \leqslant e_{c 1}$ and $\sigma_{b c}\left(e_{D}^{s}, e_{V}^{s}\right)=\sigma_{c 1}\left(r_{D V}\right) \exp \left[\left(-e_{V}^{s}-e_{c 1}\right) H_{c}\left(r_{D V}\right) / \sigma_{c 1}\left(r_{D V}\right)\right]$ otherwise, where $r_{D V}=\left|e_{D}^{s}\right| / e_{V}^{s}$ for $e_{V}^{s}>0$ and $r_{D V}=-\left|e_{D}^{s}\right| /\left(e_{V}^{s}-e_{V 0}^{s}\right)$ for $e_{V}^{s} \leqslant 0$ in which $e_{V 0}^{s}=\kappa_{c 3} e_{c 0}, e_{c 0}=\sigma_{c 0} / E_{0}, \sigma_{c 0}$ is the mesoscale yielding compressive stress, $e_{c 1}=\kappa_{c 0} e_{c 0}$ is the strain at which rehardening starts, $\kappa_{c 0}$ and $\kappa_{c 3}$ are model parameters, and $\sigma_{c 1}\left(r_{D V}\right)=\sigma_{c 0}+\left(e_{c 1}-e_{c 0}\right) H_{c}\left(r_{D V}\right)$. The function $H_{c}\left(r_{D V}\right)$ is written as $H_{c}\left(r_{D V}\right)=H_{c 1}+\left(H_{c 0}-H_{c 1}\right) /\left(1+\kappa_{c 2}\left\langle r_{D V}-\kappa_{c 1}\right\rangle\right)$ where $H_{c 0}, H_{c 1}, \kappa_{c 1}$ and $\kappa_{c 2}$ are model parameters. In most cases, $H_{c 0}=$ $0.4 E_{0}$ and $\kappa_{c 3}=0.1[? ?]$.

The frictional behavior due to compression-shear (again for $e_{N}^{s}<0$ ) is formulated to capture the increase in the shear strength under confined compression. The incremental shear stress in $\mathbf{m}$ and $\mathbf{l}$ directions are computed as $\dot{t}_{M}^{s}=E_{T}\left(\dot{e}_{M}^{s}-\dot{e}_{M}^{s p}\right), \dot{t}_{L}^{s}=E_{T}\left(\dot{e}_{L}^{s}-\dot{e}_{L}^{s p}\right)$ where $\dot{e}_{M}^{s p}=\dot{\lambda} \partial U_{p} / \partial t_{M}^{s}$, $\dot{e}_{L}^{s p}=\dot{\lambda} \partial U_{p} / \partial t_{L}^{s}, \lambda$ is the plastic multiplier and $U_{p}$ is the plastic potential defined as $U_{p}=\left(t_{M}^{s 2}+t_{L}^{s 2}\right)^{1 / 2}$. The yieling surface is defined as $\varphi_{p}=\sigma_{T}-\sigma_{b s}$ where the shear yielding stress $\sigma_{b s}$ is a nonlinear frictional law written as $\sigma_{b s}\left(t_{N}^{s}\right)=\sigma_{s}+\left(\mu_{0}-\mu_{\infty}\right) \sigma_{N 0}\left[1-\exp \left(t_{N}^{s} / \sigma_{N 0}\right)\right]-\mu_{\infty} t_{N}^{s}$ where $\mu_{0}$ and $\mu_{\infty}=0$ are the initial and final internal friction coefficients and $\sigma_{N 0}$ is the normal stress which corresponds to the transition from $\mu_{0}$ to $\mu_{\infty}$.

One of the major impact of hight temperature on concrete is the thermal degradation due to chemical and physical reactions that occur at elevated temperatures [? ? ? ? ]. Exposure to high temperatures may cause considerable variations in the physical and mechanical properties with irreversible 
loss of strength and stiffness, and increased ductility in the post-peak regime [? ? ? ? ? ]

Abundant experimental data available in the literature [? ? ? ? ? ? ] gives a general understanding of thermal degradation. The elastic modulus decreases up to $20 \%$ of its initial value when concrete is heated to $800{ }^{\circ} \mathrm{C}$ (see in Fig. 3 (a)). The compressive strength changes slightly (depending upon on aggregate type) before $400{ }^{\circ} \mathrm{C}$, and has a $80 \%$ decrease from 400 to $800{ }^{\circ} \mathrm{C}$ (see in Fig. 3 (b)). The tensile splitting strength decreases to $20 \%$ of its initial value at $800{ }^{\circ} \mathrm{C}$ (see in Fig. 3 (c)). Similarly the direct tensile strength has a $70 \%$ reduction when heated to $600{ }^{\circ} \mathrm{C}$. The macroscale fracture energy $\left(G_{F}\right)$ generally has a $60 \%$ increase at $300{ }^{\circ} \mathrm{C}$ and it decreases for temperatures large than $300{ }^{\circ} \mathrm{C}$. It is worth pointing out that the experimental data plotted in Fig. 3 is relevant only to ordinary Portland cement concrete with conventional aggregate such as siliceous gravel, sandstone, and limestone.

Thermal degradation on the mesoscopic facet in LDPM is introduced through the following function:

$$
f_{d}=1-\frac{\exp \left(n_{d}\right) \Theta}{1-\Theta\left(1-\exp \left(n_{d}\right)\right)}
$$

where $n_{d}$ is the parameter controlling the shape of the thermal degradation evolution and $\Theta$ is a temperature level variable defined as:

$$
\Theta=\frac{\left\langle T-T_{s}\right\rangle}{T_{m}-T_{s}}
$$

where $T_{s}$ is the temperature at which concrete starts to degrade and $T_{m}$ is the temperature at which concrete starts to melt. LDPM material parameters, which govern the mesoscale behavior, namely $\sigma_{t}$ (tensile strength), $\sigma_{s}$ (shear strength), $E_{0}$ (effective normal elastic modulus), $\sigma_{c 0}$ (yielding compressive 
stress) and $\sigma_{N 0}$ (transitional stress), are assumed to decay proportional to $f_{d}$ with the parameters identified from experimental data. The tensile characteristic length $\left(l_{t}\right)$ and all the other is assumed to remain constant.

Since the chemical and physical reactions are not explicitly considered as individual thermal strain components, the calibrations and verifications of various experiments are necessary for the correctness of the parameters in thermal degradation formula. In this study, the unconfined uniaxial compression tests [? ], triaxial compression tests [? ], and tensile strength tests [? ] were simulated. The calibration results are plotted in Fig. 3 and the degradation parameters are reported in Tab. 1.

It is worth mentioning that with the thermal degradation parameters in Tab. 1 the macroscale fracture energy computed by three-pointing bending tests fits the experimental data automatically [? ? ? ? ] as shown in Fig. 3 (e). This confirms the assumption that the tensile characteristic length is temperature independent.

Table 1: Thermal degradation of LDPM parameters

\begin{tabular}{|c|c|c|c|c|}
\hline \multicolumn{2}{|c|}{ Parameters } & $T_{s}$ & $T_{m}$ & $n_{d}$ \\
\hline \multicolumn{2}{|c|}{ Unit } & {$\left[{ }^{\circ} \mathrm{C}\right]$} & {$\left[{ }^{\circ} \mathrm{C}\right]$} & {$[-]$} \\
\hline$\sigma_{t}$ & Tensile strength & 274 & 1000 & 0.4 \\
\hline$\sigma_{s}$ & Shear strength & 274 & 1000 & 0.4 \\
\hline$E_{0}$ & Effective normal elastic modulus & 20 & 1000 & 0.7 \\
\hline$\sigma_{c 0}$ & Compression yielding stress & 340 & 1000 & 0.2 \\
\hline$\sigma_{N 0}$ & Transitional stress & 340 & 1000 & 0.2 \\
\hline
\end{tabular}

At high temperature, when fluid (water and/or vapor) fills with the pores 

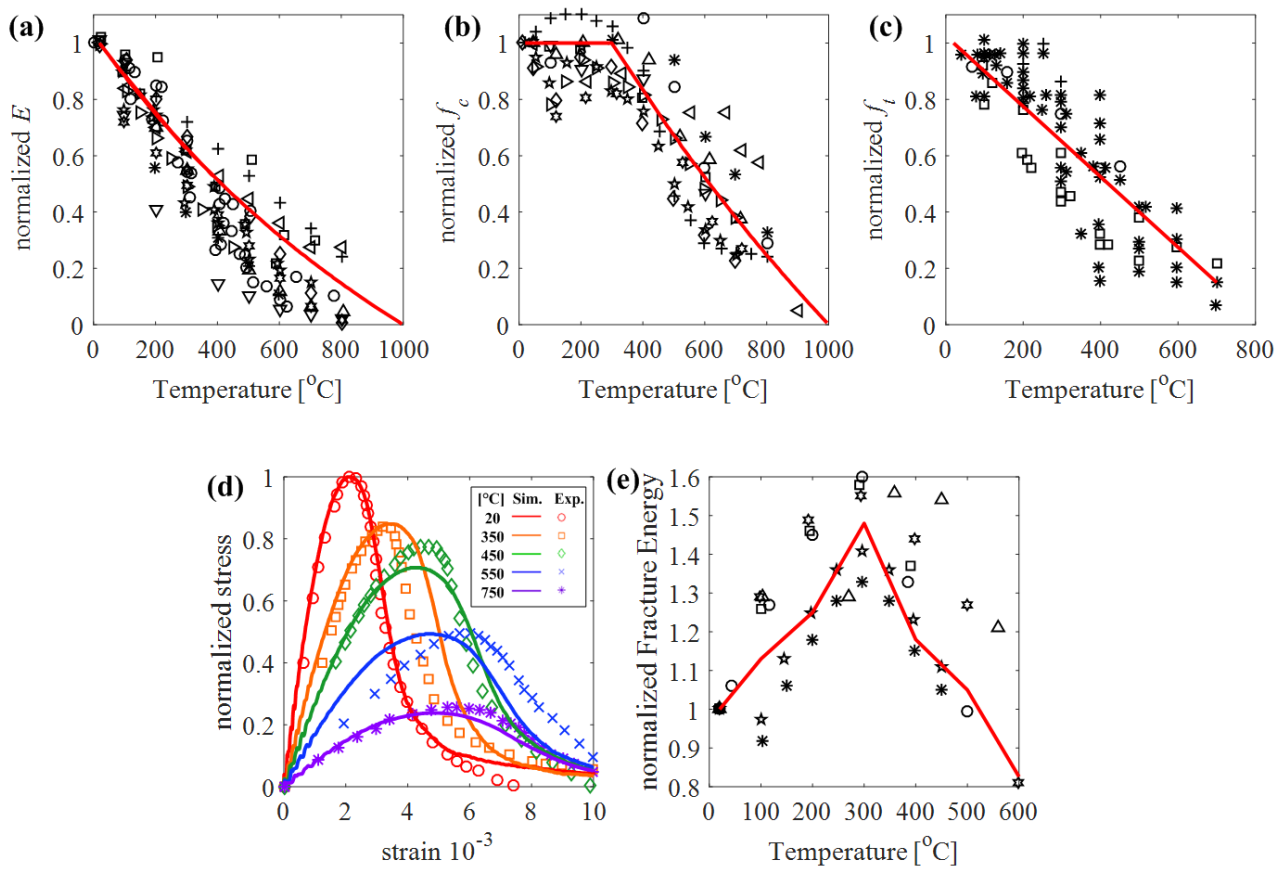

Figure 3: LDPM at high temperature [? ? ? ? ? ? ]. (a) Thermal effect on elastic modulus. (b) Thermal effect on compressive strength. (c) Thermal effect on tensile strength. (e) Uniaxial compression stress vs. strain curves [? ]. (f) Thermal effect on fracture energy [? ? ? ? ? ].

and cracks, the whole cell system is regarded as a multiphase porous material with solid skeloton and fluid, and the cell is still a homogeneous medium. The internal stress of solid skeloton $\left(\mathbf{t}^{s}\right)$ is not only caused by the deformation but also by the pressure $(p)$ of water or vapor. Hence the total stress vector on each facet can be computed as:

$$
\mathbf{t}=\mathbf{t}^{s}-b \mathbf{t}^{w}
$$

where, $b$ is the Biot coefficient [? ? $], \mathbf{t}^{w}=p \mathbf{n}$ is the average pore fluid (liquid or vapor) pressure vector with $p>0$ is the pore pressure computed by 
the DTemPor3 model described later. In the current formulation, the Biot coefficient is assumed to be equal to 1 unless otherwise mentioned [? ].

The mesoscopic crack opening vector associated with each facet of the LDPM meso-structure can be calculated as:

$$
\boldsymbol{\delta}=\delta_{N} \mathbf{n}+\delta_{L} \mathbf{l}+\delta_{M} \mathbf{m}
$$

where $\delta_{N}=l\left(e_{N}^{s}-t_{N}^{s} / E_{N}\right), \delta_{L}=l\left(e_{L}^{s}-t_{L}^{s} / E_{L}\right), \delta_{M}=l\left(e_{M}^{s}-t_{M}^{s} / E_{M}\right) ; \delta_{N}$ is its normal component, and $\delta_{M}$ and $\delta_{L}$ are two shear components related to the sliding of crack surfaces.

Finally, the LDPM governing equations are completed through the force and moment equilibrium of each LDPM cell:

$$
\sum_{k \in \mathcal{F}_{I}} A_{k}^{p} \mathbf{t}_{k}+V_{I} \mathbf{b}=\mathbf{0}, \quad \sum_{k \in \mathcal{F}_{I}} A_{k}^{p} \mathbf{c}_{k} \times \mathbf{t}_{k}=\mathbf{0}
$$

where $\mathcal{F}_{I}$ is the set containing all the facets of a generic polyhedral cell $I$, $A_{k}^{p}=A_{k} \mathbf{n}^{\mathrm{T}} \mathbf{n}_{k}$ is the area of the projected facet $k[?]$ where $\mathbf{n}$ is the orientation of the tetrahedron edge associated to the facet $k$ and $\mathbf{n}_{k}$ is the unit vector orthogonal to the facet $k$ with area $A_{k}, \mathbf{c}_{k}$ is the vector representing the distance between the centroid of the facet $k$ and the center of the cell, $V_{I}$ is the cell volume and $\mathbf{b}$ is the external body forces applied to the cell. The computational framework is implemented into the MARS code [?] and solved with an explicit dynamic solver.

\subsection{The Hygro-Thermal model (DTemPor3)}

Considering the discrete character of LDPM, the hygro-thermal equations are formulated with reference to a three-dimensional network of onedimensional elements anchored to the LDPM system [? ? ]. The formulation adopted hereinafter is an extension of the two-dimensional continuum 
formulation proposed by Bažant and coworkers [? ], to the three-dimensional discrete settings. The new formulation is entitled DTemPor3.

\subsubsection{Flow Lattice Element System}

For the sake of clarity, the construction of the Flow Lattice Element (FLE) system is explained in 2D. Let's consider two points inside two adjacent LDPM tetrahedra. They are labeled as node " $\mathrm{T}_{1}$ " and " $\mathrm{T}_{2}$ " in the $2 \mathrm{D}$ representation of Fig. 4 (a) and they are selected to coincide with the Tetpoint of the LDPM tessellation [? ]. The segment connecting $\mathrm{T}_{1}$ and $\mathrm{T}_{2}$ is called "FLE ${ }_{12}$ ". Each FLE has an associated volume as shown in Fig. 4 (a) in 2D. Each tetrahedron is characterized by four FLEs governing the mass transport and heat transfer from and to all adjacent tetrahedra.

In $3 \mathrm{D}$ the $\mathrm{FLE}_{12}$ domain volume $\left(V_{w}\right)$ consists of two pyramids, termed "Side $\mathrm{T}_{1} "\left(V_{w 1}\right)$ and "Side $\mathrm{T}_{2} "\left(V_{w 2}\right)$. The length of $\mathrm{FLE}_{12}$ is $l_{12}$ which can be divided into two segments by the intersection with the tetrahedron face $\mathrm{P}_{1} \mathrm{P}_{2} \mathrm{P}_{3}$ (Fig. $4(\mathrm{~b})$ ). The two segment lengths $l_{1}$ and $l_{2}$ can be defined by the length proportionality coefficients $g_{1}$ and $g_{2}$ which satisfy the relations $l_{i}=g_{i} l_{12}$. The superscript $i=1,2$ identify variables relevant to the Side $T_{1}$ and the Side $\mathrm{T}_{2}$, respectively. In Fig. 4 (b), a is the unit vector orthogonal to the surface $\mathrm{P}_{1} \mathrm{P}_{2} \mathrm{P}_{3}$, and $\mathbf{e}$ is the direction of FLE $\mathrm{E}_{12}$ from $\mathrm{T}_{2}$ to $\mathrm{T}_{1}$. The projection of the area $\mathrm{P}_{1} \mathrm{P}_{2} \mathrm{P}_{3}\left(A_{\triangle}\right)$ in the e direction is defined as $A_{w}=$ $-A_{\triangle} \mathbf{a}^{\mathrm{T}} \mathbf{e}$. One can write $V_{w}=V_{w 1}+V_{w 2}=l_{12} A_{w} / 3$.

The common geometry features of LDPM Facets and FLEs allow coupling the mechanical behavior, particularly fracturing, with the transport phenomena. As illustrated in Fig. 4 (c), each FLE domain volume contains 6 LDPM facets and half of them are on each side of the FLE base. When 

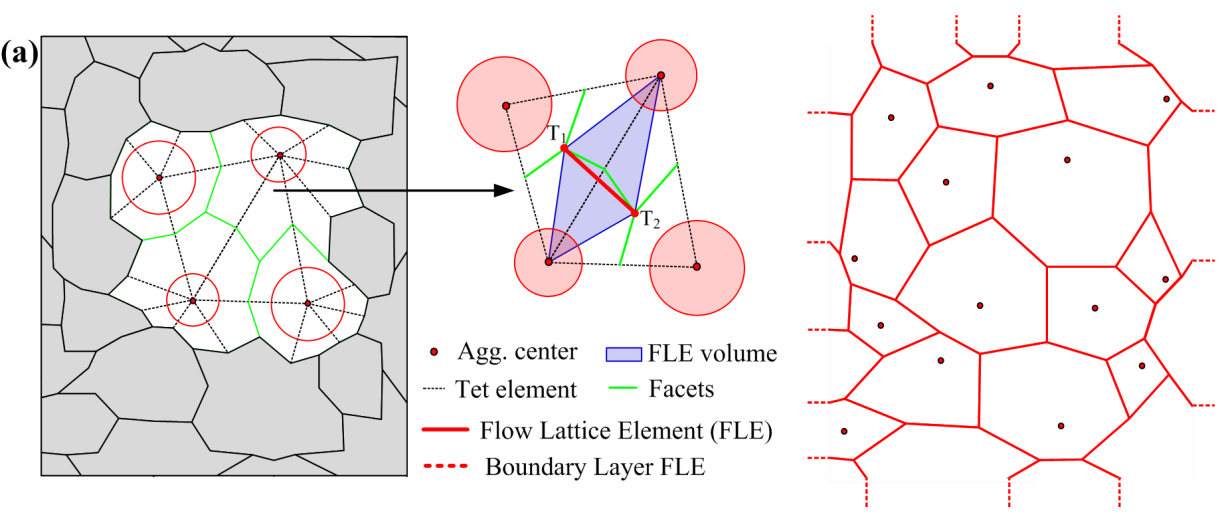

(b)

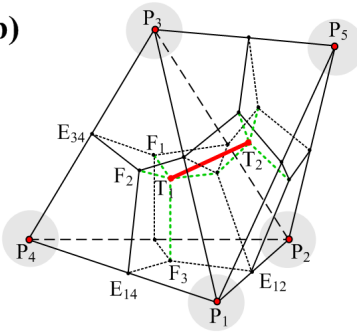

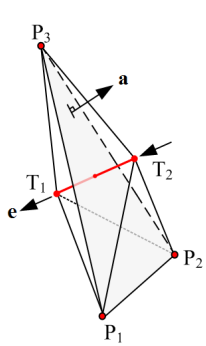

(d)

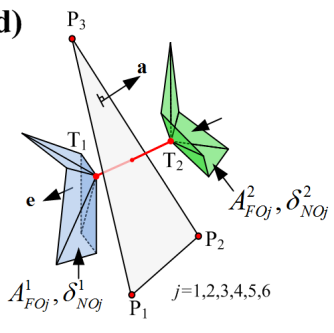

Figure 4: Flow Lattice Element (FLE) system geometry. (a) FLE generation in 2D. (b) FLE in 3D. (c) LDPM facets related to mass transport. (d) LDPM facets related to heat transfer.

concrete fractures the domain volume of the $\mathrm{FLE}_{12}$ becomes the sum of the crack space at the facets $\left(V_{c}\right.$ with a crack area, $A_{c}$, on the $\left.\mathrm{P}_{1} \mathrm{P}_{2} \mathrm{P}_{3}\right)$ and the uncracked material $\left(V_{w}\right)$. The total crack volume can be calculated as $V_{c}=\sum_{i=1}^{2} \sum_{j=1}^{3}\left(\delta_{N P j}^{i} A_{F P j}^{i}\right)$ in which $\delta_{N P j}^{i}$ denotes the normal crack opening of a facet connecting with $\mathrm{T}_{i}$ and $A_{F P j}^{i}$ is the corresponding facet area.

The pore pressure and temperature on LDPM Tetrahedral nodes " $\mathrm{T}_{1}$ " and " $\mathrm{T}_{2}$ " represent the mass thermodynamic state in the $\mathrm{FLE}_{12}$ domain volume. The fluxes through $\mathrm{FLE}_{12}$ represent the mass and heat exchange between two adjacent LDPM Tetrahedra. Accordingly, for each FLE, the 
mass and heat balance equations can be written as:

$$
\frac{d}{d t}\left(V_{w i} w_{w}+V_{c i} w_{c}\right)=A_{w}\left(D_{1} \frac{p_{2}-p_{1}}{l_{12}}+D_{2} \frac{T_{2}-T_{1}}{l_{12}}\right)
$$

$$
\frac{d}{d t}\left(V_{w i} U_{w}+V_{c i} U_{c}\right)=A_{w}\left(D_{3} \frac{p_{2}-p_{1}}{l_{12}}+D_{4} \frac{T_{2}-T_{1}}{l_{12}}\right)
$$

where, $t$ is time; $p_{i}(t)$ and $T_{i}(t)$ are pore pressure and temperature at $T_{i}$, $(i=1,2) ; w_{w}(p, T)$ and $U_{w}(p, T)$ denote the water mass and heat content per unit volume of uncracked material (subscript " $w$ "); $w_{c}(p, T)$ and $U_{c}(p, T)$ represent water mass and heat content per unit volume of cracked material (subscript "c"); $D_{1}$ is the hydraulic conductivity (permeability) in Fick's Law and $D_{3}$ is the thermal conductivity in Fourier's Law; $D_{2}$ and $D_{4}$ are the coefficient for Soret mass flux and Dufour heat flux, $D_{1}, D_{2}, D_{3}$ and $D_{4}$ are the effective properties that must take into accrount the effect of cracking as explained later in this paper. The uncracked material volume $V_{w i}$ and their common section area $A_{w}$ are considered to be constant since the deformation of the uncracked material is negligible compared to the area increase due to cracking. Hence the domain volume increase of each FLE is assumed to come from the initiation and propagation of cracks on the corresponding facets $\left(V_{c i}\right)$ and the elastic volume increase of $V_{w}$ is neglected.

By defining the weighted average pressure and temperature, $p=\sum_{i=1}^{2}\left(g_{i} p_{i}\right)$ and $T=\sum_{i=1}^{2}\left(g_{i} T_{i}\right)$, to represent the overall state of $\mathrm{FLE}_{12}$, the governing equations for $\mathrm{FLE}_{12}$ can be written as:

$$
\mathbf{M} \dot{\mathbf{u}}+\mathbf{K u}=\mathbf{S}
$$


where,

329

$$
\mathbf{M}=V_{w}\left[\begin{array}{cccc}
g_{1} C_{1} & g_{1} C_{2} & 0 & 0 \\
g_{1} C_{3} & g_{1} C_{4} & 0 & 0 \\
0 & 0 & g_{2} C_{1} & g_{2} C_{2} \\
0 & 0 & g_{2} C_{3} & g_{2} C_{4}
\end{array}\right]
$$

330

$$
\mathbf{K}=\frac{A_{w}}{l_{12}}\left[\begin{array}{cccc}
D_{1} & D_{2} & -D_{1} & -D_{2} \\
D_{3} & D_{4} & -D_{3} & -D_{4} \\
-D_{1} & -D_{2} & D_{1} & D_{2} \\
-D_{3} & -D_{4} & D_{3} & D_{4}
\end{array}\right]
$$

331

$$
\mathbf{S}=V_{w}\left[\begin{array}{lllll}
g_{1} s_{1} & g_{1} s_{2} & g_{2} s_{1} & g_{2} s_{2}
\end{array}\right]^{\mathrm{T}}
$$

$$
\mathbf{u}=\left[\begin{array}{llll}
p_{1} & T_{1} & p_{2} & T_{2}
\end{array}\right]^{\mathrm{T}}
$$

332 in which, $C_{1}=\partial w_{w} / \partial p+V_{c} / V_{w} \partial w_{c} / \partial p ; C_{2}=\partial w_{w} / \partial T+V_{c} / V_{w} \partial w_{c} / \partial T$; $C_{3}=\partial U_{w} / \partial p+V_{c} / V_{w} \partial U_{c} / \partial p ; C_{4}=\partial U_{w} / \partial T+V_{c} / V_{w} \partial U_{c} / \partial T ; s_{1}=$ ${ }_{334}-w_{c} \dot{V}_{c} / V_{w}$ and $s_{2}=-U_{c} \dot{V}_{c} / V_{w}$.

335 It is also worth pointing out that the source term $s_{1}=-w_{c} \dot{V}_{c} / V_{w}$ governs the pressure release due to cracking behavior. The contribution of water dehydration is reflected in the term $-\partial w_{d} / \partial T$ in $C_{2}$ on the left side of the mass balance equation.

\subsubsection{Initial and boundary conditions}

The variables of state in the presented model are pore pressure, $p$, and absolute temperature, $T$. For the initial condition, the pore pressure, $p_{0}=$ 
$h_{0} p_{\text {sat }}\left(T_{0}\right)$, is defined by the initial relative humidity, $h_{0}$, and the initial temperature, $T_{0}$, of the concrete volume under investigation.

As a first approximation, mass and heat flux from the concrete surface to the environment may be considered to be linearly dependent on the difference of surface pore pressure and environmental pressure, and the difference of surface temperature and environmental temperature, respectively.

A so-called "boundary layer" (see in Fig. 4(a)) consisting of FLEs orthogonal outward to the concrete surface with constant cross section and lengths $l_{b p}$ for pressure and $l_{b T}$ for temperature is added to the FLE system to simulate the linear drop between the state variables at the specimen surface and the environment. All boundary layer FLEs are assumed to have the same material parameters of the FLEs which share the nodes on the specimen surface. In this way, the boundary conditions applied on the boundary layer can be simplified to Dirichlet type conditions, $T_{b o}=T_{e n}$, and $p_{b o}=p_{e n}$, where, $T_{b o}$ and $p_{b o}$ are temperature and pore pressure of the extended boundary layer nodes; $T_{e n}$ is the temperature of the environment; $p_{e n}=h_{e n} p_{s a t}\left(T_{0}\right)$ is the pressure of the environment, which can be calculated by the environmental relative humidity $h_{e n}$. By varying the length of the boundary layer, one can simulate various degrees of surface emissivity. In this study, $l_{b p}=l_{b T}=1$ mm was used.

\subsubsection{Water content}

The water in the concrete pores can be subdivided into several components, capillary water, physically absorbed water, hindered water in nanopores, water vapor and chemically bound water. Capillary water exists as liquid water in large pores. In smaller pores and walls of large pores at low relative 
humidity, a considerable amount of water is absorbed on the solid skeleton surfaces. In nanopores, the absorbed water layer cannot fully developed and it becomes hindered, that is water with much smaller mobility than capillary and absorbed water. In partially saturated pores, all the types of water can be consider to be in thermodynamic equilibrium with water vapor. Since all the water types but the chemically bounded can be removed during heating at $105{ }^{\circ} \mathrm{C}$, they are collectively known as evaporable water. The chemically bound water is known as nonevaporable water, because this kind of water is a part of the solid skeleton and it can be removed only upon cement dehydration at very high temperatures.

According to this classification and considering old enough concrete for which hydration might be assumed to be nearby complete, the water content per unit volume of concrete in the uncracked material can be divided into evaporable water $\left(w_{e}\right)$, and water released by dehydration $\left(w_{d}\right)$ at high temperature:

$$
w_{w}=w_{e}-w_{d}
$$

in which, the evaporable water content, $w_{e}(T, p)$, is a function of temperature and pore pressure, and the water release due to dehydration, $w_{d}(T)$, is a function of temperature. It is worth pointing out that, in some works [? ? ], the dehydration water is included as source term which should appear on the right side of mass balance equations in Eq. 9. In the present model, the dehydration water is included on the left side of the balance equations as a negative term following Ref. [? ].

The desorption isotherms are equilibrium curves of pressure versus specific water content at constant temperature. The semi-empirical isotherm 
presented by Bažant et al. [? ] is widely accepted in the literature [? ? ? ] and it is adopted in this work as well. The water content in concrete at high temperature can be expressed as:

$$
w_{e}=\left\{\begin{array}{lll}
c\left(\frac{w_{0}}{c} h\right)^{1 / m_{\text {iso }}} & \text { if } & h \leqslant 0.96 \\
w_{e}^{1.04}-\frac{w_{e}^{1.04}-w_{e}^{0.96}}{1.04-0.96}(1.04-h) & \text { if } & 0.96<h<1.04 \\
w_{f}[1+0.12(h-1.04)] & \text { if } & h \geq 1.04
\end{array}\right.
$$

in which, $c$ is the cement content per unit volume of concrete; $w_{0}$ is the water content per unit volume of saturated concrete at $25{ }^{\circ} \mathrm{C}$; $w_{f}$ denotes the water content per unit volume of saturated concrete at any temperature, $w_{f}=w_{0}+w_{d} ; m_{\text {iso }}(T)$ is a temperature dependent coefficient given by:

$$
m_{\text {iso }}(T)=1.04-\frac{\left(T_{C}+10\right)^{2}}{\left(T_{C}+10\right)^{2}+22.3(25+10)^{2}}
$$

Based on the original assumption in TemPor, the relative humidity is defined to represent the water status in pores which is expressed as:

$$
h=\frac{p}{p_{\text {sat }}(T)} \approx S^{m_{\text {iso }}}=\left(\frac{w_{w}}{w_{f}}\right)^{m_{\text {iso }}}
$$

in which, $S$ is the saturation. When temperature is lower than the water critical temperature, $T_{C r i t}=375.15{ }^{\circ} \mathrm{C}$, the pressure at saturation can be calculated as [?]:

$$
p_{\text {sat }}=\sum_{i=1}^{7} a_{i}^{c} T^{i}
$$

where $a_{1}^{c}=-1.15466360 \cdot 10^{6} \mathrm{~Pa} /{ }^{\circ} \mathrm{C}, a_{2}^{c}=3.2147795276 \cdot 10^{4} \mathrm{~Pa} /{ }^{\circ} \mathrm{C}^{2}, a_{3}^{c}=$ $1.591032529 \mathrm{~Pa} /{ }^{\circ} \mathrm{C}^{3}, a_{4}^{c}=-3.258874385 \cdot 10^{2} \mathrm{~Pa} /{ }^{\circ} \mathrm{C}^{4}, a_{5}^{c}=-3.92808082 \cdot$ $10^{-5} \mathrm{~Pa} /{ }^{\circ} \mathrm{C}^{5}, a_{6}^{c}=4.424390583 \cdot 10^{-6} \mathrm{~Pa} /{ }^{\circ} \mathrm{C}^{6}$, and $a_{7}^{c}=-1.43742222 \cdot$ 
$10^{-9} \mathrm{~Pa} /{ }^{\circ} \mathrm{C}^{7}$. If $T>T_{\text {Crit }}$, liquid water does not exist at any pressure can one can assume $p_{\text {sat }}=p_{\text {sat }}\left(T_{\text {Crit }}\right)$. Hence, one need to remember that the $h$ can be larger than 1 in our numerical model which physically represents the over-saturated status in pores.

In the above isotherm (Eq. 17), the pore water state can be non-saturated ( $h \leqslant 0.96)$, saturated and over-saturated $(h \geq 1.04)$ and transitional $(0.96<$ $h<1.04)$. The case of $0.96<h<1.04$ represents the vapor condensation or liquid water evaporation processes. In the case of $h \geq 1.04$, the water content can be larger than that at saturation at any temperature $\left(w_{e}>w_{f}\right)$. This is justified by the increase in concrete porosity due to elastic deformation and cracking behavior which provide more space for the water.

The nonevaporable water, which is chemically bound within cement hydrated products is gradually released in the pores for temperatures ranging from $105^{\circ} \mathrm{C}$ to $1000^{\circ} \mathrm{C}$. Dehydration is a complex phenomenon that depends on many factors, including but not limited to, temperature history, hydration degree, and concrete mix design. In this work, the empirical equation proposed by Gawin et al. [? ] is used to describe this process:

$$
w_{d}=\left\{\begin{array}{lll}
0 & \text { if } & T \leqslant 105^{\circ} \mathrm{C} \\
0.32 \alpha_{c}^{\infty} c f_{w d}\left(T_{\max }(t)\right) & \text { if } & T>105^{\circ} \mathrm{C}
\end{array}\right.
$$

in which, $\alpha_{c}^{\infty}=1.032 w_{m i x} / c /\left(0.194+w_{m i x} / c\right)$ is the asymptotic hydration degree [? ? ]; $w_{m i x}$ is the initial water content in the concrete mix; the term $0.32 \alpha_{c}^{\infty} c$ represents the maximum mass of water per unit volume of concrete that can be released during dehydration; $T_{\max }$ is the highest temperature reached by concrete during heating; $f_{w d}(T)$ is a dimensionless function of 
temperature with the following expression:

$$
f_{w d}(T)=\sum_{i=1}^{3} b_{i}^{c}(T-105)^{i}
$$

where, $b_{1}^{c}=1.7151 \cdot 10^{-3}, b_{2}^{c}=-4.0006 \cdot 10^{-7}$ and $b_{3}^{c}=-2.9507 \cdot 10^{-10}$ are empirical coefficients with unit $1 /{ }^{\circ} \mathrm{C}$.

As soon as fractures occur in the control domain, water diffusion is driven by pressure gradient in the cracks. Assuming that the crack volume is filled with water, the mass of water per unit volume of crack is $w_{c}=\rho_{c}$, where $\rho_{c}$ will be the vapor-liquid mixture density in the cracks which is be discussed later.

\subsubsection{Permeability}

Neglecting the relatively rather small contribution due to temperature gradient $\left(D_{2}=0\right.$ in Eq. 9), the water flow in the uncracked material can be simplified as Darcy diffusion process.

Concrete permeability to water transport is affected by the porosity and its distribution, and the saturation level of the pores. The former is directly controlled by many factors, such as mix composition, curing, temperature degradation, and damage; the latter is related to the relative humidity in the pores. Hence the permeability of the uncracked material, $D_{w}$, can be formulated by following Bažant and coworkers [? ] as:

$$
D_{w}=f_{1}(h) f_{2}(T) D_{w}^{0}
$$

where $D_{w}^{0}$ is the initial permeability. $f_{1}(h)$ is a relative humidity dependent 
447

function with the following expression:

$$
f_{1}(h)=\left\{\begin{array}{lll}
1 & \text { if } & h>1 \\
\alpha^{\prime}+\frac{1-\alpha^{\prime}}{1+\left(\frac{1-h}{1-h_{c}}\right)^{4}} & \text { if } & h<1
\end{array}\right.
$$

448

in which $\alpha^{\prime}=1 /\left[1+0.253\left(100-\min \left(T_{C}, 100\right)\right)\right], h_{c}=0.75$, and $f_{2}(T)$ is a temperature depend function defined as:

$$
f_{2}(T)=10^{C_{T}\left(T-T_{0}\right)}
$$

in which $C_{T}$ is a factor that accounts for the increase of permeability in the uncracked concrete at elevated temperature ranging from 0.0025 to 0.005 [? ? ] and $T_{0}$ (in $\left.\mathrm{K}\right)$ is the initial temperature.

Damage and cracking have a large influence on water transport because they increase the overall permeability and, as consequence, they induce a decrease in the pore pressure. Water is more likely to flow through damaged material (cracks) than through the uncracked material and then cracks become important paths for seepage. The water flow in the cracks can be formulated by postulating a Poiseuille type of flow which corresponds to the following cracked permeability [? ]:

$$
D_{c}^{i}=\frac{\rho_{c}}{12 \mu_{c}} \frac{1}{A_{w}} \sum_{j=1}^{3} l_{N P j}\left(\delta_{N P j}^{i}\right)^{3}
$$

in which, $\mu_{c}$ is the dynamic viscosity of the vapor-liquid mixture in cracks; $\delta_{N P j}^{i}$ denotes the facet normal crack openings on side $\mathrm{T}_{i}(i=1,2)$ in the FLE domain, $j=1,2,3 ; l_{N P j}$ is the crack length on the $\mathrm{P}_{1} \mathrm{P}_{2} \mathrm{P}_{3}$ surface (see in Fig. 4(c)). 
Although the crack opening of facets on each side in the FLE domain is generally different, the mass flux through connecting cracks should be exactly the same. Hence the mass flux in cracks is assumed to follow a series model:

$$
D_{c}=\left(\frac{g_{1}}{D_{c}^{1}}+\frac{g_{2}}{D_{c}^{2}}\right)^{-1}
$$

Furthermore, assuming the flows in uncracked material and cracks have no interaction in the FLE domain, the effective permeability of a single FLE (Eq. 13) can be computed by the following parallel model:

$$
D_{1}=D_{e f f}=D_{w}+D_{c}
$$

\subsubsection{Heat capacity}

The heat capacity is the specific heat multiplied by the density and it is a non-linear function of temperature and pressure. The latent heat effects are incorporated as a part of the water phase changes, chemically bound water dehydration, and evaporation-condensation. The phase change of $\alpha-\beta$ inversion of quartz and the chemical change of carbonation-decarbonation are not explicitly introduced in the formulation. Therefore, the thermal energy per unit volume of concrete can be expressed as the algebraic sum of the heat density in the uncracked material, the heat consumption due to dehydration process and latent heat:

$$
U_{w}=U_{s}-U_{d}-U_{v}
$$

where the heat density in uncracked material can be expressed as $U_{s}=\rho_{s} C_{s} T$ with the solid skeleton density $\rho_{s}=2400 \mathrm{~kg} / \mathrm{m}^{3}$, and the heat capacity $C_{s}=900+80\left(T_{C} / 120\right)-4\left(T_{C} / 120\right)^{2}[?]$. The dehydration heat consumption 
is defined as $U_{d}=C_{d} w_{d}$, in which $C_{d}=2400 \mathrm{~J} / \mathrm{kg}$ is the heat consumption of dehydration per kilogram of concrete [? ]. The term $U_{v}=w_{e} H_{l}$ represents the heat consumption of evaporation and condensation in which $H_{l}$ represents the enthalpy of liquid water as a function of $p$ and $T$, and $w_{e}$ is the amount of evaporable water per unit concrete. This term is typically neglectable largely due to the fact that water constitutes a small portion of the mass of concrete [? ].

The heat energy of the vapor-liquid water mixture in the unit volume of cracks can be expressed as $U_{c}=\rho_{c} C_{c} T$, where $C_{c}$ is the heat capacity of the vapor-liquid water mixture in the cracks. By taking the time derivative, one can obtains $\dot{U}_{c}=\rho_{c} C_{c} \dot{T}+T \dot{h} \partial\left(\rho_{c} C_{c}\right) / \partial h$, in which $\partial\left(\rho_{c} C_{c}\right) / \partial h \approx 0$ as in most cases the states of water (liquid or vapor) is stable.

The coefficients of heat transfer in Eq. 11 can be then calculated as: $C_{4}=\rho_{s} C_{s}-C_{d} \partial w_{d} / \partial T+\rho_{c} C_{c} V_{c} / V$. The source term, $s_{2}=-\rho_{c} C_{c} T \dot{V}_{c} / V$, is obtained due to the thermal exchange between the uncracked material and the vapor-liquid water mixture in the cracks.

\subsubsection{Heat conductivity}

Since the thermal exchange caused by pressure gradient is quite small it is usually ignored $\left(D_{3}=0\right)$. Hence the transient heat transport can be reduced to Fourier's Law in which $D_{4}$ physically represents the effective thermal conductivity.

As emphasized in the recent studies by Shen et al. [? ? ? ], it is the cracks sub-orthogonal to thermal conduction, rather than the sub-parallel cracks, that have profound negative effect on concrete heat conduction performance. For this reason, the crack opening of 12 facets sub-orthogonal to 
FLE (Fig. 4(d)) are used to take into account the effect of fracture on the thermal conduction by using the following series model:

$$
D_{4}=\lambda_{e f f}=\left(1+\frac{A_{c}}{A_{w}}\right)\left(\frac{l_{12}}{\lambda_{w}^{0}}+\frac{\bar{\delta}_{N O}^{1}}{\lambda_{c}}+\frac{\bar{\delta}_{N O}^{2}}{\lambda_{c}}\right)^{-1}\left(l_{12}+\bar{\delta}_{N O}^{1}+\bar{\delta}_{N O}^{2}\right)
$$

in which, $\lambda_{w}^{0}$ is the initial thermal conductivity of uncracked material; $\bar{\delta}_{N O}^{i}=$ $\sum_{j=1}^{6} \delta_{N O j}^{i} / 6(i=1,2)$ is the average normal cracking opening of the 6 facets sub-orthogonal to FLE on each side $\mathrm{T}_{i} ; \lambda_{c}$ is the thermal conductivity of the vapor-liquid water mixture in the cracks, and $1+A_{c} / A_{w} \approx 1$.

\subsubsection{Effect of water evaporation and condensation in the cracks}

To determine $\rho_{c}, C_{c}, \lambda_{c}$ and $\mu_{c}$ in the cracks, an important issue is to determine the water state, because condensation and evaporation of free water might happen. The water states in the cracks is assumed to depend on the relative humidity. If $h>1.1$, water in the cracks is liquid, $\chi_{c}=\chi_{l}$. When $h<1$, the water state is vapor, $\chi_{c}=\chi_{v}$. Otherwise, when $1<h \leqslant 1.1$, water is a mixture of liquid water and vapor. The smoothstep function is employed to model the phase change:

$$
\chi_{c}=\left(\chi_{l}-\chi_{v}\right)\left\{-2[10(h-1)]^{3}+3[10(h-1)]^{2}\right\}+\chi_{v}
$$

in which, $\chi_{c}, \chi_{v}$ and $\chi_{l}$ are parameters for the vapor-liquid mixture, vapor and liquid water, respectively. The same function $\chi_{c}$ is used for $\rho_{c}, C_{c}$, $\lambda_{c}$, and $\mu_{c}$, and $\rho_{l}=1000 \mathrm{~kg} / \mathrm{m}^{3}, \rho_{v}=0.6 \mathrm{~kg} / \mathrm{m}^{3}, C_{l}=2 \mathrm{~kJ} /\left(\mathrm{kg} \cdot{ }^{\circ} \mathrm{C}\right)$ , $C_{v}=4.17 \mathrm{~kJ} /\left(\mathrm{kg} \cdot{ }^{\circ} \mathrm{C}\right), \lambda_{l}=0.6 \mathrm{~W} /\left(\mathrm{m} \cdot{ }^{\circ} \mathrm{C}\right), \lambda_{v}=0.0265 \mathrm{~W} /\left(\mathrm{m} \cdot{ }^{\circ} \mathrm{C}\right)$, $\mu_{l}=800 \times 10^{-6} \mathrm{~Pa} \cdot \mathrm{s}, \mu_{v}=12 \times 10^{-6} \mathrm{~Pa} \cdot \mathrm{s}[? ?]$. 


\subsection{Two-way coupling scheme}

The hygro-thermo-mechanical coupled problem of concrete thermal spalling here is enforced as a two-way coupling solution of the LDPM-HT (Section 2.1) and the DTemPor3 model (Section 2.2). While the LDPM Cells system provides a mesoscopic description of concrete mechanical behavior and the FLEs network serves as a source of mechanical loads caused by the temperature gradient and built-up pore pressure, the dual-lattice system allows for the coupled simulation of the heat transfer, mass transport and cracking behavior of concrete in the spatial dimension.

However, the difficulty locates at the mismatch of time steps between the LDPM-HT and the DTemPor3 model. The LDPM-HT uses an explicit dynamic algorithm with a central difference [? ], while the time integration of the DTemPor3 model is performed by means of the Crank-Nicolson (implicit) method [? ]. Hence in the time dimension, a staggered coupling scheme is proposed to bridge the gap between the simulation time step $\left(t^{s}\right)$ in the explicit solver for LDPM-HT and the real time step $\left(t^{r}\right)$ in the implicit method for DTemPor3 model.

The numerical implementation of the two-way coupling scheme is illustrated in Fig. ??. At the selected simulation time step, the LDPM-HT makes use of the solutions ( $T$ and $p$ ) from the DTemPor3 model at the corresponding real time step, whereas properties for the DTemPor3 model are updated by using the information from the LDPM-HT (crack opening). But one need to notice that the a simulation time step generally does not have its one-toone correspondence of real time step. Hence a simulation real time $\left(r t^{s}\right)$ is mapped from the simulation time step and a linear interpolation is applied 
to compute the thermal and hydraulic loads for the LDPM-HT solution at the corresponding simulation time step. In turn, a new step of the DTemPor3 model $\left(t^{r}\right)$ uses the crack opening distribution from LDPM-HT at the nearest $t^{s} \longrightarrow r t^{s}<t^{r}$.

The staggered coupling scheme along with the mixed explicit-implicit integration scheme provides a simple yet efficient approach for two-way coupling simulations of the hygro-thermo-mechanical coupled problem. The proposed framework of the Multi-physics Lattice Discrete Partial Model (MLDPM) is implemented into the MARS software [? ], which is a structural analysis computer code with an object-oriented architecture that makes the implementation of new computational technologies very effective.

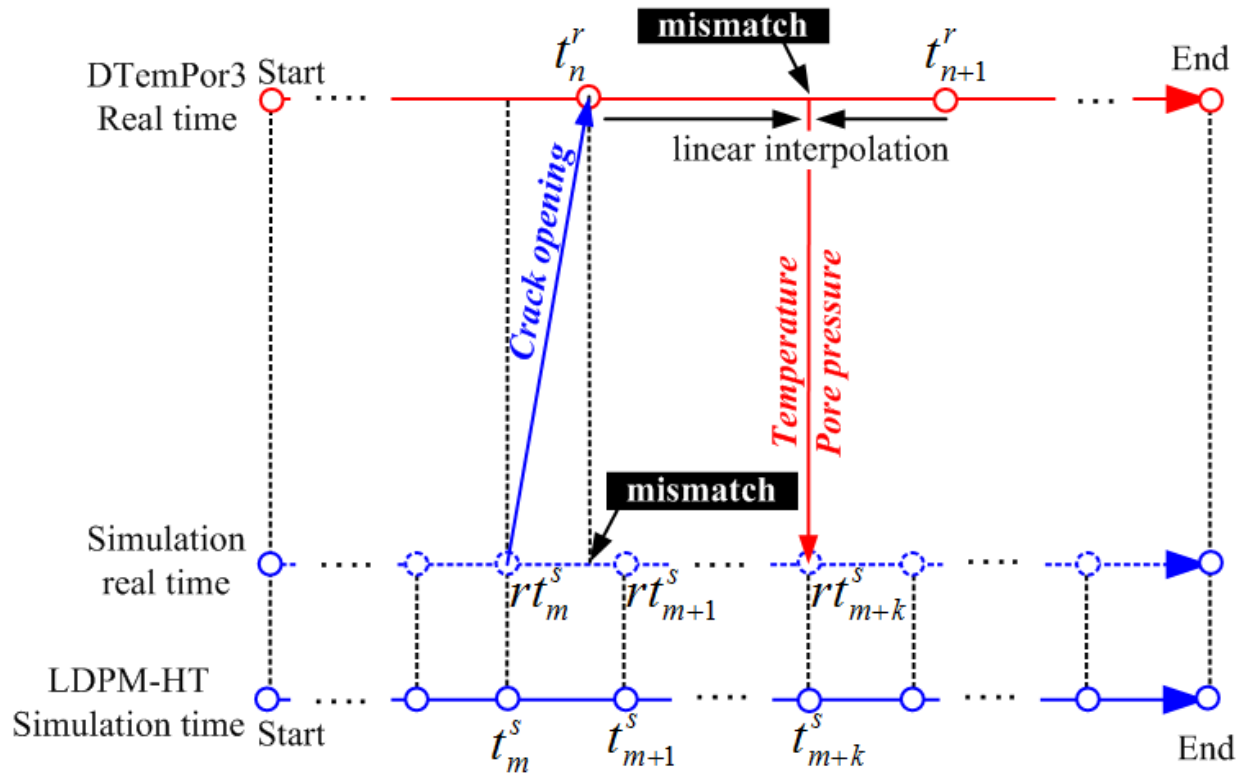

Figure 5: Illustration of the two-way coupling scheme. $t_{m+k}^{s}>t_{m+1}^{s}>t_{m}^{s}>0$ are the simulation steps and their corresponding simulation real time steps are $r t_{m+k}^{s}>r t_{m+1}^{s}>$ $r t_{m}^{s} \cdot t_{n+1}^{r}>t_{n}^{r}>0$ are the real time steps. $m, n, k$ are natural numbers. 


\section{Numerical Example}

\subsection{Experiment and simulation setup}

The effectiveness of the coupled LDPM-DTemPor3 model is here demonstrated by simulating a set of high temperature experiments reported by Kalifa et al. [? ]. As illustrated in Fig. ??(a), the experiments are relevant to concrete slabs with a thickness of $0.12 \mathrm{~m}$ and in-plane dimensions of $0.3 \mathrm{~m}$ $\times 0.3 \mathrm{~m}$. They were exposed to the environment at their top and bottom surfaces and thermally insulated on all lateral sides. From their initial storage conditions, the slabs were heated to $600{ }^{\circ} \mathrm{C}$ at their top surface for a period of 6 hours. Two types of concrete were tested: high-performance concrete (HPC) and ordinary concrete (OC) characterized by the mix-designs reported in Table. ??. Throughout these tests, the internal pressure and temperature in the central part of the slabs were measured by sensors at different depth from the heated face as shown in Fig. ??(a).

In the numerical analyses the size of the slabs was reduced to save computational cost. The simulated slab had the actual thickness of $120 \mathrm{~mm}$ and the in-plane dimensions of $150 \mathrm{~mm} \times 150 \mathrm{~mm}$. The heating and cooling loads $\left(T_{e n}\right)$ measured in the experiments were applied at the top and bottom surfaces, respectively, and the environmental relative humidity $\left(h_{e n}\right)$ was assumed to be 0.6. The initial condition of the specimen was characterized by $T_{0}=25{ }^{\circ} \mathrm{C}$ and $h_{0}=0.95$. From the simulations, data on nine nodes at every $10 \mathrm{~mm}$ depth were output (Fig. ??(a)). The lateral boundaries were sealed to heat transfer and mass transport and were mechanically supported only allowing the thermal expansion through the specimen depth.

It is important to point out that, in the real experiments, the lateral 
sides were not perfectly sealed. This can cause some degree of discrepancy between the numerical and experimental results as it will be discussed later. However, this approximation was adopted by all numerical studies available in the literature because it is virtually impossible to determine the actual condition [? ? ? ? ].

All parameters for HPC and OC used in the numerical analyses are listed in Tab. ?? which were carefully identified to match the experimental results. The size of aggregate particles, ranging from 4 to $20 \mathrm{~mm}$, and the mix designs used for the LDPM mesh generation were chosen on the basis of the actual concrete mix used in Kalifa et al.'s experiment [? ].

\subsection{Numerical simulation of $H P C$ slabs}

The numerical results obtained by the DTemPor3 model and the experimental data for HPC are presented in Fig. ??.

Fig. ??(b) shows the heating and cooling curves which represent the thermal loads applied at the two ends of the slab. One can see that the numerical temperature results (solid lines) are in good agreement with the experimental data (dots) at the temperature from 25 to $250{ }^{\circ} \mathrm{C}$, while a certain mismatch appear after 3 hours of heating. This seems to be caused by the difference in boundary condition, because in the experiments the lateral sides of the specimens were not perfectly sealed as in the numerical simulations.

Fig. ??(c) shows the relation between pore pressure and temperature. It is clear that the rising parts of the pore pressure curves at various depth agrees with the saturated vapor pressure curve (black dashed curve). This is also supported by the observation in the experiments [? ]. In turn, this also proves the rationality to ignore the contribution of dry air in the pore pressure 
Table 2: Concrete mix-designs and model parameters used in the simulations

\begin{tabular}{|c|c|c|c|c|c|}
\hline Parameters & Unit & Definition & $\mathrm{OC}$ & $\mathrm{HPC}$ & Source \\
\hline \multicolumn{6}{|l|}{ Mix-designs } \\
\hline$c$ & {$\left[\mathrm{~kg} / \mathrm{m}^{3}\right]$} & Cement content & 350 & 377 & {$[?]$} \\
\hline$w_{\operatorname{mix}} / c$ & {$[-]$} & Water/cement ratio & 0.5 & 0.34 & {$[?]$} \\
\hline$a / c$ & {$[-]$} & Aggregate/cement ratio & 5.23 & 5.09 & {$[?]$} \\
\hline$d_{a}$ & {$[\mathrm{~mm}]$} & Aggregate maximum diameter & 20 & 20 & {$[?]$} \\
\hline \multicolumn{6}{|c|}{ LDPM at room temperature } \\
\hline$E_{0}$ & {$[\mathrm{MPa}]$} & Normal Modulus & 43748 & 62500 & Identified \\
\hline$\sigma_{t}$ & {$[\mathrm{MPa}]$} & Tensile strength & 4 & 5 & Identified \\
\hline$l_{t}$ & {$[\mathrm{~mm}]$} & Tensile characteristic length & 120 & 80 & Identified \\
\hline$\sigma_{s}$ & {$[\mathrm{MPa}]$} & Shear strength & 10.8 & 30 & Identified \\
\hline$\sigma_{c 0}$ & {$[\mathrm{MPa}]$} & Compressive yielding stress & 150 & 200 & Identified \\
\hline$\mu_{0}$ & {$[-]$} & Initial friction & 0.2 & 0.4 & Identified \\
\hline$\sigma_{N 0}$ & {$[\mathrm{MPa}]$} & Transitional stress & 600 & 300 & Identified \\
\hline$\kappa_{c 0}$ & {$[-]$} & Strain limit ratio & 2 & 5 & {$[? ?]$} \\
\hline$\kappa_{c 1}$ & {$[-]$} & Vol. dev. damage threshold ratio & 2 & 1 & {$[? ?]$} \\
\hline$\kappa_{c 2}$ & {$[-]$} & Vol. dev. damage magnitude & 1 & 2 & {$[? ?]$} \\
\hline$d_{0}$ & {$[\mathrm{~mm}]$} & LDPM minimum diameter & 4 & 4 & Assumed \\
\hline \multicolumn{6}{|c|}{ DTemPor3 model } \\
\hline$w_{0}$ & {$\left[\mathrm{~kg} / \mathrm{m}^{3}\right]$} & Initial water content & 100 & 100 & {$[?]$} \\
\hline$D_{w}^{0}$ & {$[\mathrm{~m} / \mathrm{s}]$} & Initial permeability & $8 \times 10^{-13}$ & $5 \times 10^{-13}$ & Identified \\
\hline$\lambda_{w}^{0}$ & {$\left[\mathrm{~W} /\left({ }^{\circ} \mathrm{C} \cdot \mathrm{m}\right)\right]$} & Initial thermal conductivity & 2 & 2.2 & Identified \\
\hline$C_{T}$ & {$[-]$} & Temp. effect on permeability & 0.0045 & 0.004 & Identified \\
\hline
\end{tabular}


(a)

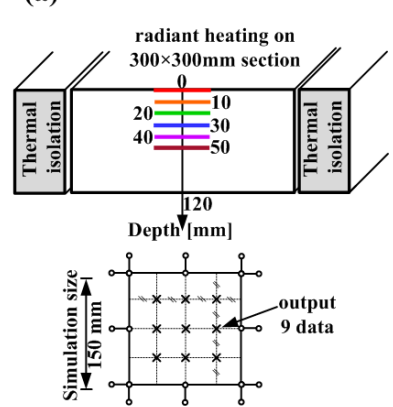

(d)
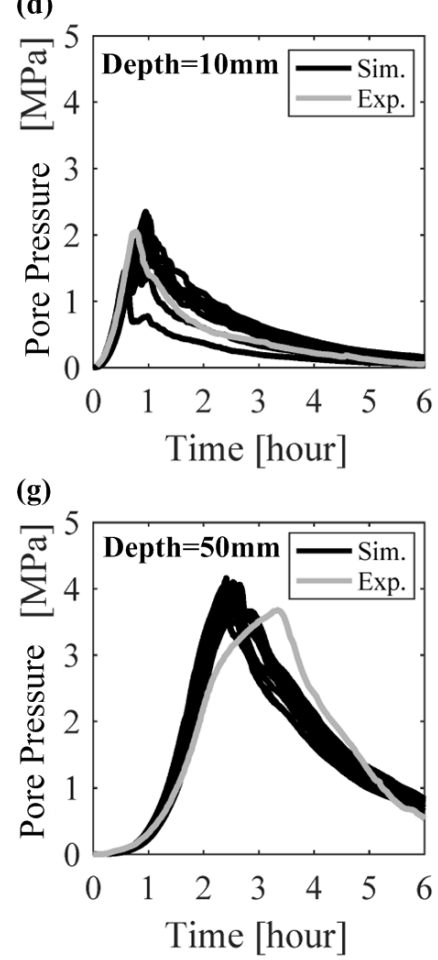

(b)

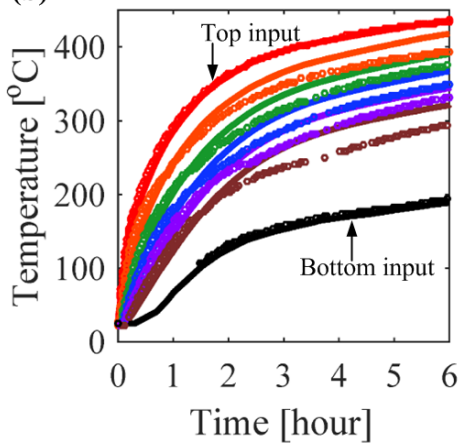

(e)

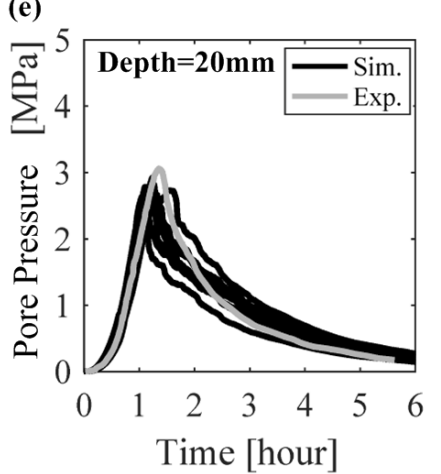

(h)

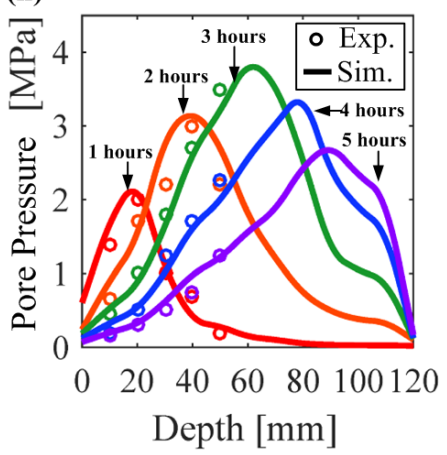

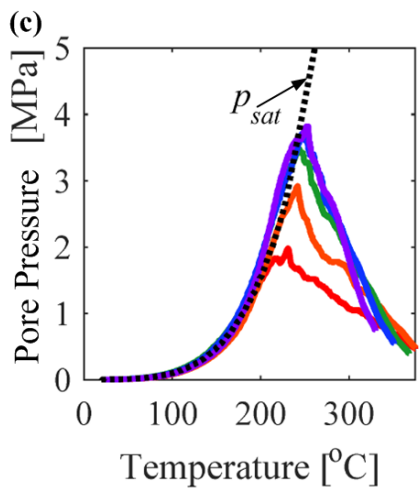

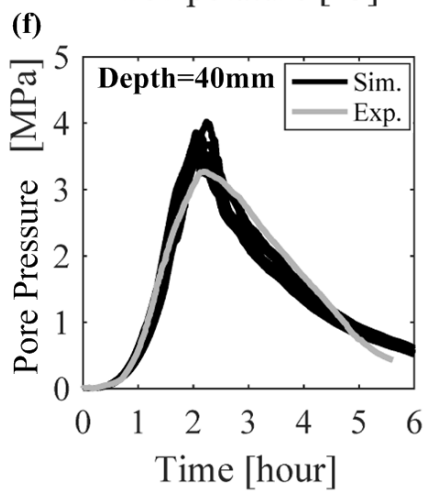

(i)

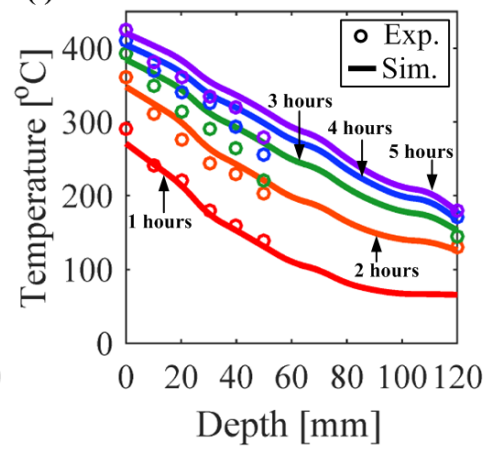

Figure 6: Comparison of numerical and experimental results for HPC. (a) Test setup. (b) Temperature evolution with time. (c) Pressure evolution with temperature. (d-g) Pressure evolution with time at 10, 20, 40 and $50 \mathrm{~mm}$ depth. (h) Pressure evolution with depth. (i) Temperature evolution with depth. 
in the DTemPor3 model. The built-up pressure peak keeps increasing with the depth and its value never exceeds $4 \mathrm{MPa}$.

In Fig. ??(d-h) one can see that the DTemPor3 model reproduces the experimentally recorded pressure values very well both in magnitude and profile shape. The peak value at a depth of $50 \mathrm{~mm}$ (Fig. ??(h)) occurs a little earlier in the simulations than in the experiments. This may be caused by the already mentioned difference in pressure boundary conditions between experiments and simulations. Indeed, in the simulations, due to the perfect sealing of the lateral surface more water is retained inside the slab leading to an earlier and higher pressure peak.

Fig. ??(h) and (i) show pressure and temperature distributions along the depth at different times after heating. The pressure peak moves inward as the heating time increases. The peak value increases along the depth close to the heated surface and then decreases close to the bottom. The pressure peak value approaches the maximum value of $4 \mathrm{MPa}$ at the slab mid-depth.

Fig. ?? illustrates the contours of $T, p, S, D_{\text {eff }}, \lambda_{\text {eff }}$ and total crack opening after a 3-hour of heating. Vapor with high pressure moves to low pressure zones driven by the pressure gradient. Part of the water mass escapes from the specimen at the heating surface and the rest moves to a lower temperature zone, where vapor condensates into liquid water forming a saturated zone next to the peak pressure area. This phenomenon is known in the literature as "moisture clog" [? ]. Fig. ??(d) and (e) show the $D_{\text {eff }}$ and $\lambda_{\text {eff }}$ at a certain time step. The evolution of $D_{\text {eff }}$ and $\lambda_{\text {eff }}$ is due to the combined effect of crack opening, pressure and temperature. 
(a) Temperature $\left[{ }^{\circ} \mathrm{C}\right]$

(b) Pore pressure $[\mathrm{MPa}]$

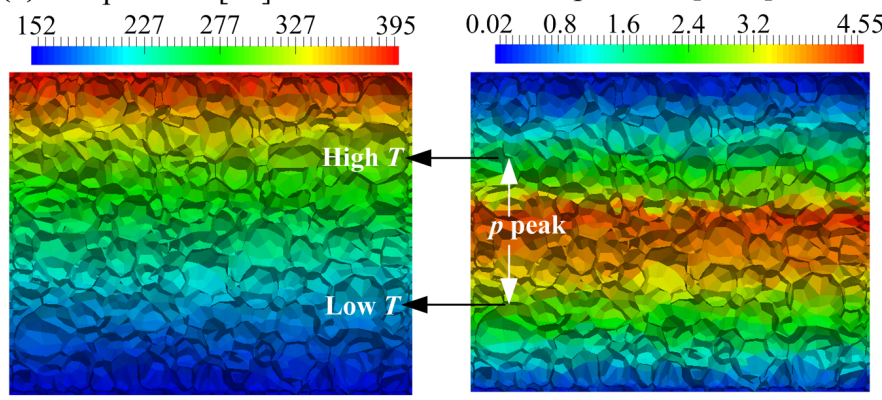

(c) Saturation [-]

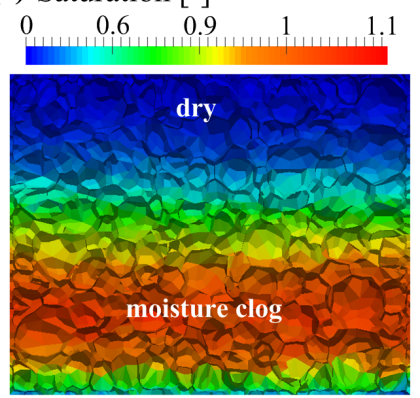

(d) $D_{\text {eff }}[\mathrm{m} / \mathrm{s}]$

$5 \times 10^{-13} 6.3 \times 10^{-12} 4 \times 10^{-11} 2.5 \times 10^{-10} \quad 5 \times 10^{-9}$

(e) $\lambda_{\text {eff }}[\mathrm{W} /(\mathrm{K} \cdot \mathrm{m})]$
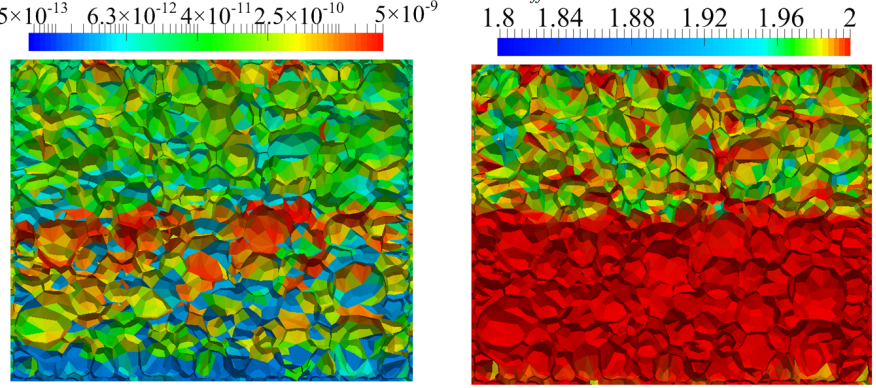

(f) Total crack opening [mm] $\begin{array}{llllll}0.001 & 0.01 & 0.03 & 0.04 & 0.063\end{array}$

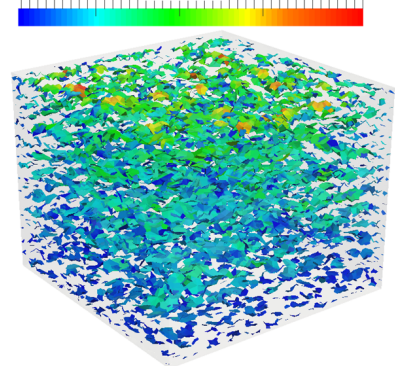

Figure 7: Results for the HPC slabs after 3 hours of heating.

637

638

\subsection{Numerical simulation of $O C$ slabs}

The same numerical analysis was carried out for OC slabs. Fig. ?? shows the numerical and experimental plots of temperature and pressure versus time measured at various distances from the slab top.

Fig. ??(a) shows the temperature development during heating from both the experiments (dots) and the simulations (solid lines). The heating and cooling curves are the thermal loads applied at the two ends of the slab.

Fig. ??(b) provides the relation between pressure and temperature. Differently from the HPC, the pressure peaks remain around 1.25 MPa throughout the depth. This was also recorded in the experiments and it is caused by the fact that more pore-necks [? ] become mass transport paths in OC than 
in HPC during the heating process. This is reflected in a larger value of the parameter $C_{T}$ (see Tab. ??) in Eq. 25.

Fig. ??(c-g) shows good agreements between numerical and experimental data for both profile shape and values of the pressure evolution. For the same boundary conditions issue as in the HPC case, the simulations give an earlier pressure peak inside the specimen.

Fig. ??(h) and (i) show pressure and temperature distributions along the slab depth at various times. As previously observed for the HPC case, the pressure peak moves inward as the heating time increases.

\subsection{Effect of crack opening on pressure}

In this section, the contribution of crack opening in Eq. 11 to the pressure peak is examined. The parameters of HPC in Tab. ?? are used and the pressure peak values at different depth from the numerical analyses with and without cracks are plotted in Fig. ??.

One can see that the effect of crack opening becomes significant when the depth is larger than $40 \mathrm{~mm}$. At those depths, the pressure (4 MPa) is high enough to initiate and extend the cracks. In the case where the effect of cracks is included, cracks become an important mass transport path in which water can flow to lower pressure areas. As a result, the pore pressure decreases locally.

\section{Concrete thermal spalling mechanisms}

This section analyzes the main mechanisms of concrete thermal spalling. As neither pore pressure and thermal stress can be isolated in real experiments, it is very difficult to obtain a definite experimental conclusion on 
(a)

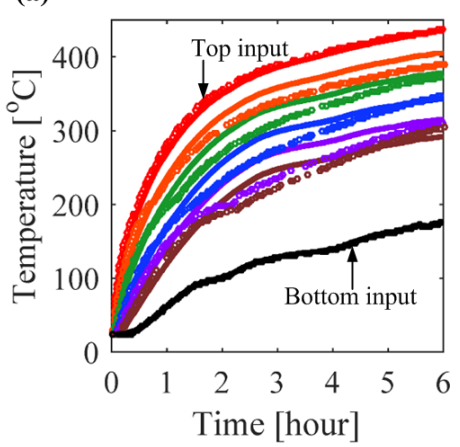

(d)

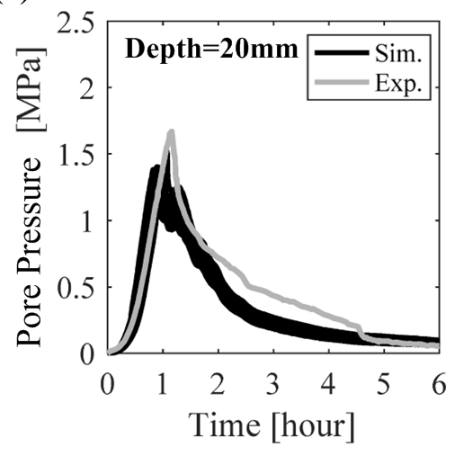

(g)

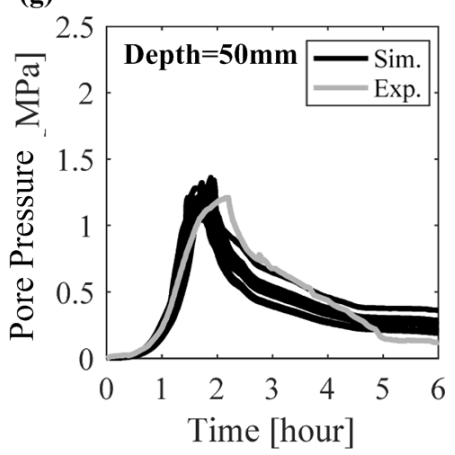

(b)

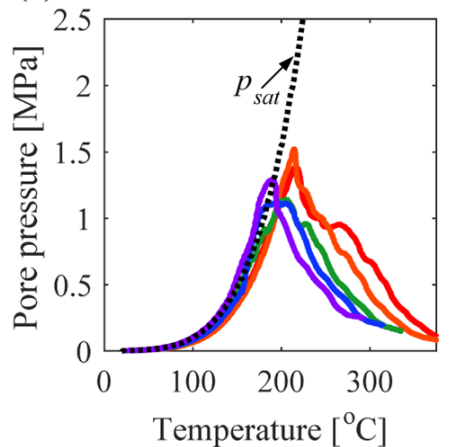

(e)

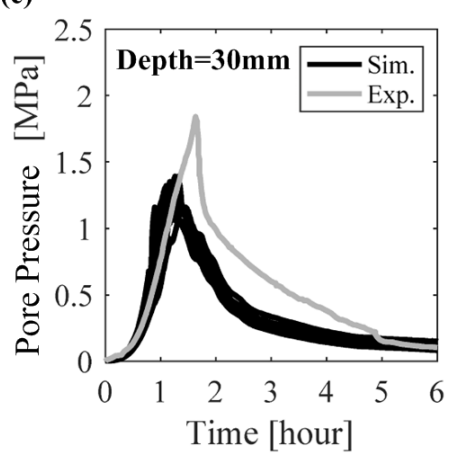

(h)

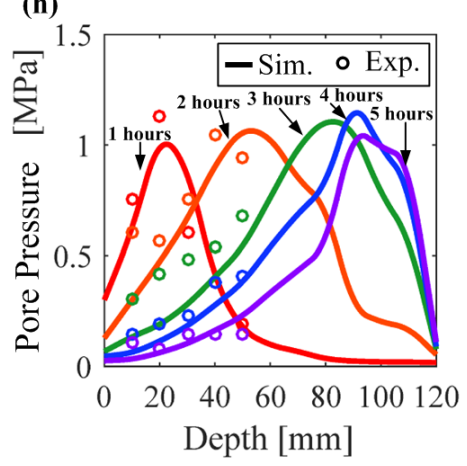

(c)

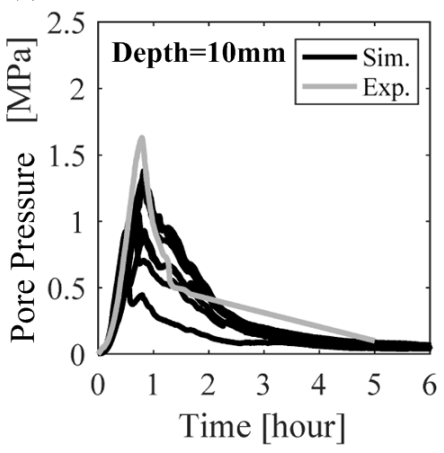

(f)

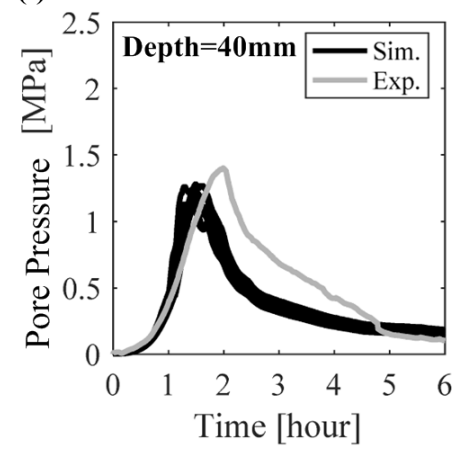

(i)

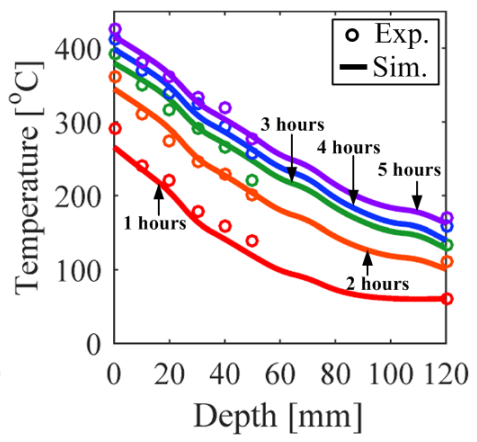

Figure 8: Comparison of numerical and experimental results for OC slabs. (a) Temperature evolution with time. (b) Pressure evolution with temperature. (c-g) Pressure evolution with time at 10, 20,30, 40 and $50 \mathrm{~mm}$ of depth. (h) Pressure evolution with depth. (i) Temperature evolution with depth. 


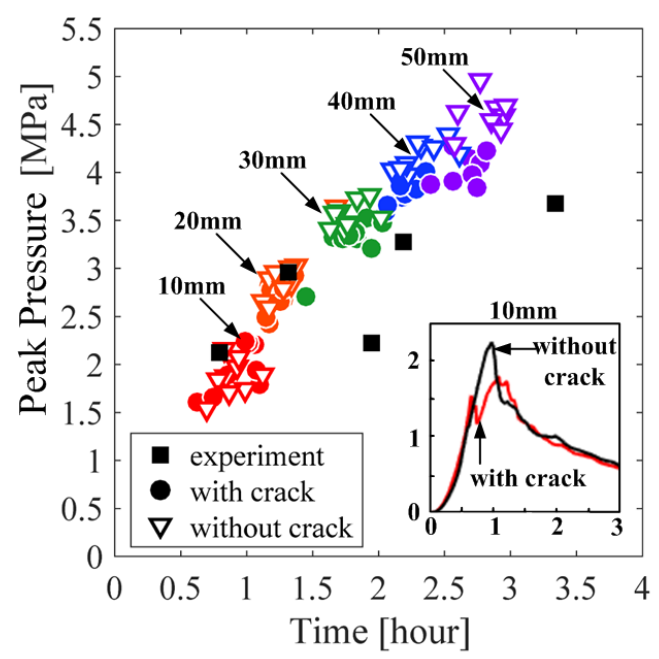

Figure 9: Effect of cracking on pressure.

which spalling mechanism is dominant. Fortunately, numerical modeling allows for each influence factor to be independently isolated to characterize the significant one and their coupling.

In this section, the considered simulations setup is the one used in the experiments carried out by Lo Monte et al. [? ]. In those tests, the specimen was a $800 \mathrm{~mm}$-side square concrete slab with a $100 \mathrm{~mm}$ thickness. The heat source, following ISO 834-1 [? ], was applied at the bottom in a central window of $600 \times 600 \mathrm{~mm}^{2}$ to keep low temperature in the peripheral $100 \mathrm{~mm}$ concrete rim experiencing confinement. Sixteen radial slits were cut in order to break the mechanical continuity. In the simulations the temperature measured at the heating surface was applied at the bottom of the slab according to the plot shown in Fig. ??

For the purpose of saving computational cost, a side length of $400 \mathrm{~mm}$ was used in the numerical analyses and, correspondingly, all other dimensions 


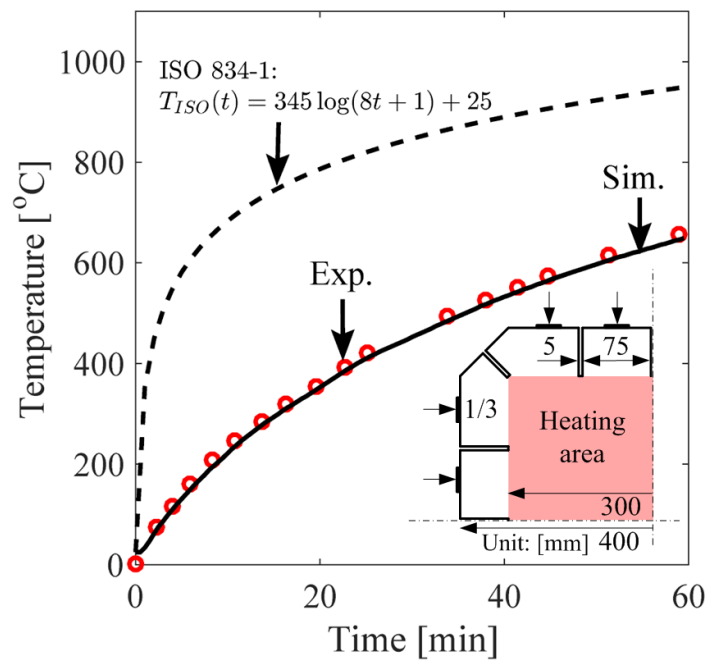

Figure 10: Spalling thermal loads ans experiment setup.

were scaled by 0.5 . The heating load (Fig. ??) was applied on the central window $(300 \times 300 \mathrm{~mm})$ and the displacements of the cold rim peripheral faces were set to zero. Since no information was available for the mechanical, flow and thermal properties of the concrete used in the experiments, the parameters for OC in Tab. ?? were used in the simulations.

First of all, the spalling phenomenon was simulated considering the effect of both thermal stresses and pressure. The pressure peak value reaches about $0.7 \mathrm{MPa}$ at 20-mm depth and the peak moves towards to the cooling surface during the heating process (Fig. ?? (a)). One need to remember that the maximum peak pressure value is much smaller than that in the simulations of Kalifa's tests. This is mainly caused by the high heating rate of ISO834. In the secondly place, the differences in concrete mix and the depth of pore pressure can influence the pore pressure build-up. The simulated temperatures fit very well with the experimental measurement along the 
depth after 10, 20 and 30 minutes of heating (Fig. ?? (b)).

Fig. ?? (a) and (b) show the crack opening distribution after 15 and 30 minutes of heating. After 15 minutes of heating, some initial spalling can be observed. As the temperature keeps increasing, the spalling area expands and many fragments are ejected from the surface. This phenomenon is similar to the high-speed camera observations obtained by Zeiml et al. [? ].

Fig. ?? (c) shows the macroscopic stress [? ] along the depth at the center of the specimen. Since the lateral surface of the cold rims is supported, the specimen is under biaxial compressive condition throughout its thickness. The absolute value of the biaxial stress reaches a maximum (50 MPa) at 16$\mathrm{mm}$ depth after 30 minutes of heating. The average pressure on the lateral surfaces of the cold rims is $21.15 \mathrm{MPa}$ after 30 minutes of heating. This value is higher than the confinement stress in the experiment, but it does not change the biaxial compressive stress status near the heating area. The spalling depth in the experiment was about $50 \mathrm{~mm}$, which is half of the slab thickness, while in the present simulation a $16 \mathrm{~mm}$ depth spalling is obtained. The most significant reason for this difference is the slab size, because the deformation in a buckling problem is significantly affected by the distance between two constrains.

The same numerical analyses were carried out again by excluding the effect of pressure ( $b=0$ in Eq. 6) or the effect of thermal expansion $\left(\alpha_{T}=0\right.$ in Eq. 2). Fig. ?? (c) shows the crack opening distribution after 30 minutes of heating if only thermal expansion is included. In this case the cracking distribution is similar to that of the complete simulation, but the spalling area is smaller and the generation of fragments is somewhat less pronounced. 

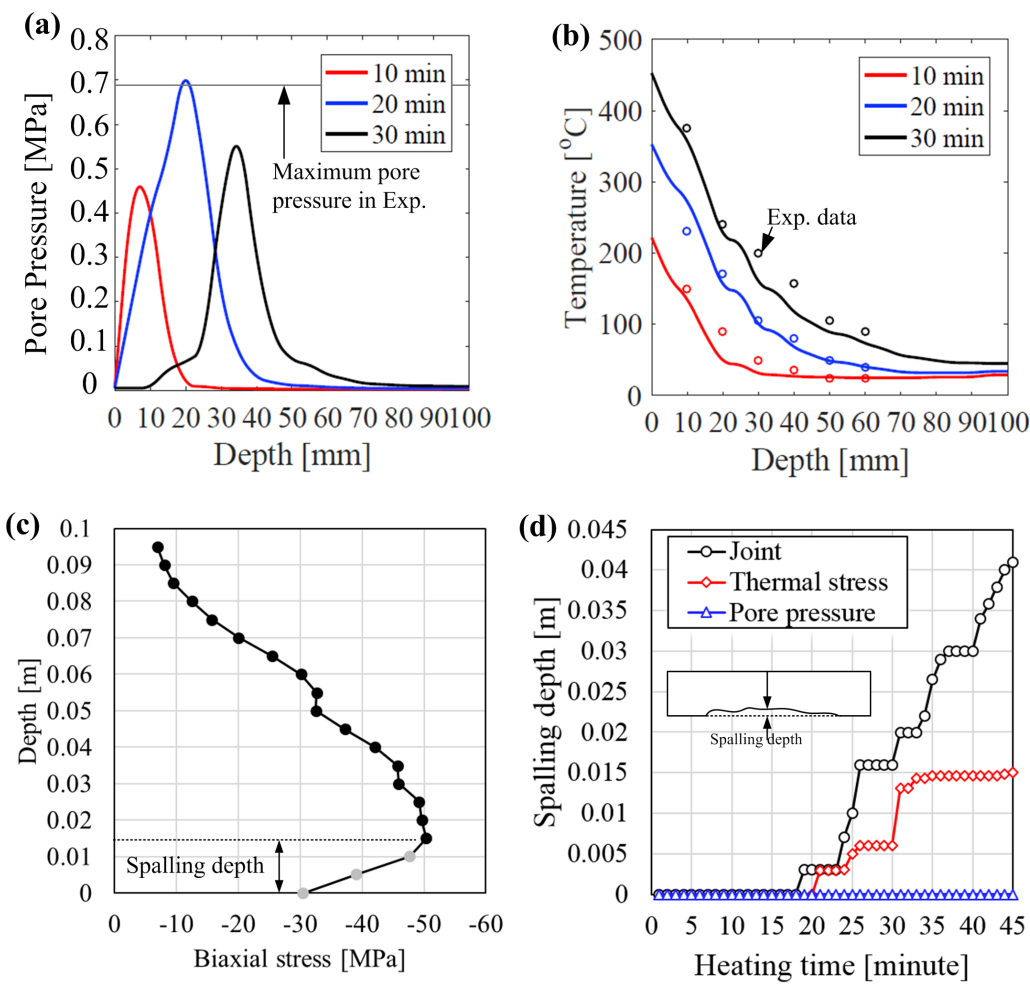

Figure 11: Results along the depth after 30 minutes of heating at the slab center $(x=$ 0,y=0): (a) Temperature, (b) Pressure, (c) Biaxial stress and (c) Spalling depth.

This indicates that thermal dilatation is a major contribution to generate damage inside the specimen and consequent spalling.

Fig. ?? (d) shows the crack opening distribution after 30 minutes of heating if only the effect of pressure is included. In this case, a totally different cracking pattern occurs. A clear macro-crack is formed near the heating surface which cannot be found in Fig. ?? (b) and much less micro-cracks are generated inside the specimen. In addition, the most important point is that no thermal spalling is observed in this case. Since thermal stresses are excluded from this simulation, much less micro-cracks appear and the pressure 


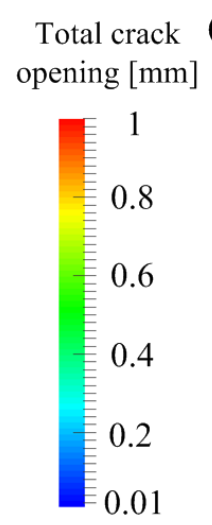

can easily build up to a high value to cause the formation of a macro-crack. However, as soon as the macro-crack appears, the increase of crack space leads to a significant reduction of pressure and no fragment can be generated. In conclusion, it is clear that the effect of pressure is not enough to cause thermal spalling, but the formation of the macro-crack isolates a layer of concrete near the surface which is then more likely to buckle and to spall if thermal stresses are present.

By comparing the three cracking pattern at 30 minutes of heating (Fig. ?? (b), (c) and (d)), one can clearly see that concrete thermal spalling is a joint action of thermal stresses and pore pressure and that thermally induced stresses play the most significant role.
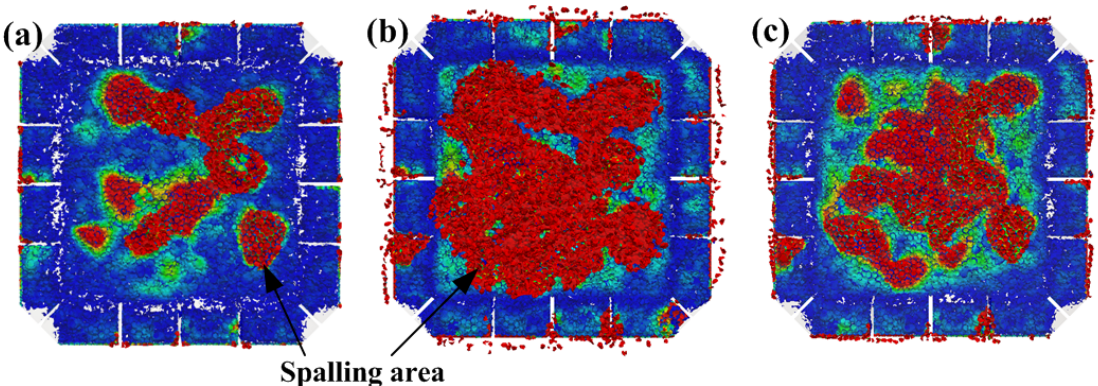

(d)
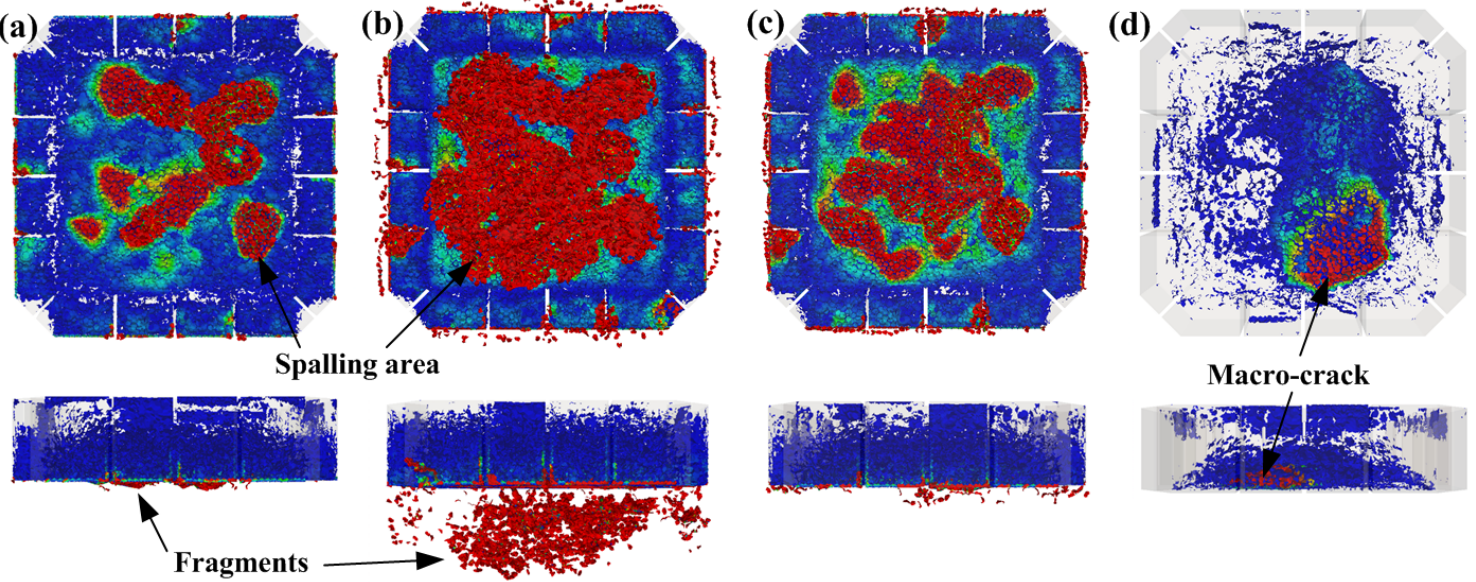

Figure 12: Crack opening contour during thermal spalling. (a) After 15 minutes of heating with all spalling factors included; (b) After 30 minutes of heating with all spalling factors included; (c) After 30 minutes of heating with only thermal stresses effect; (d) After 30 minutes of heating with only pressure effect.

The spalling depth evolutions at the center point of the slab in above cases are plotted in Fig. ?? (d). One can find that the spalling depth in 
the simulation with only thermal stress are close to that of the joint case before 25 minutes. After the heating time of 30 minutes, the spalling depth in the joint case keeps increasing while no further spalling occurs in the other cases. Therefore it is easy to understand that the contribution of thermal stress domains the spalling at the beginning and the pore pressure becomes a non-negligible factor with the increase of time/depth when/where the pore pressure can build up.

\section{Conclusions}

This study proposes a discrete three-dimensional hygro-thermal model (DTemPor3) for concrete at high temperature coupled with the Lattice Discrete Particle Model (LDPM) for the simulation of thermal spalling. The two-way coupling simulations is equipped with the capability of taking into account the effect of cracks on heat transfer and mass transport as well as the cracking behavior caused by thermal expansion and pore pressure.

Excellent agreement between model predictions and available experimental data is demonstrated by simulating both high performance concrete (HPC) and ordinary concrete (OC) slabs subject to high temperature. By using the coupled the DTemPor3-LDPM framework, the numerical simulations reproduced successfully concrete thermal spalling.

On the basis of the numerical simulations performed in this study, the following conclusions can be drawn:

(a) At elevated temperatures, the pore pressure peak in heated concrete slabs moves along the slab depth from the hot surface to the cool surface during the heating process. For HPC, the pore pressure peak value 
reaches the maximum in the middle of the slab. For OC, the pressure peak value is almost constant at various depth.

(b) Concrete fracture has a significant effect on the local pore pressure build-up and major cracks lead to a significant pressure release.

(c) Thermal stresses play a dominant role in concrete thermal spalling. Thermal expansion supplies the major contribution to generate microcracks inside concrete. Pore pressure is not enough to form spalling, although pore pressure can form macro-cracks parallel to the heat surface which are then more likely to buckle and spall under the effect of thermal strsses.

(d) Thermal spalling is obviously a joint action of pore pressure and thermal stresses. While thermal spalling is possible when thermal stresses are the only factor, spalling is much more pronounced after marcocracks are formed due to the effect of pore pressure.

(e) Thermal stress domains the spalling at the beginning, and with the increase of heating time, the pore pressure gradually becomes a nonnegligible factor when/where the pore pressure can build up.

It is disappointed that presently there is no available experimental data of the thermal degradation, the evolutions of temperature and pore pressure (low heating rate), and thermal spalling depth (high heating rate) for one concrete mix. Therefore it is very hard to accurately simulate the spalling phenomenon due to the lack of the concrete parameters in the real tests. In the further research, authors will put our efforts to build a comprehensive ex- 
794

795

796

797

perimental database and then attempt to predict the thermal spalling depth with our numerical model.

\section{Acknowledgments}

The research presented in this paper was partially supported with funds by the National Nature Science Foundation of China (51908195, 51739006) and the National Key Research and Development Program of China (2018YFC0406703). Jun Feng was supported with funds by the National Nature Science Foundation of China (11902161). The first author of this research, Lei Shen, was financially supported by the China Scholarship Council (201606710080).

This research was also supported by the QUEST high performance computing facility at Northwestern University.

\section{References}

